\title{
Temporomandibular Joint Ankylosis
}

\author{
Sonal Anchlia
}

\subsection{Introduction and Etiopathogenesis}

Temporomandibular joint (TMJ) ankylosis is defined as bony or fibrous adhesion of the anatomic joint components accompanied by limitation of mouth opening, causing difficulty in mastication, speech, and oral hygiene. This may also influence symmetry of the facial skeleton, especially in cases which occur when patient is still in the growth phase [1].

According to Kaban [2], trauma is the most common cause (31-98\%), followed by local or systemic infection (10-49\%), and lastly any systemic disease (10\%). Infection occurring in the joint commonly occurs due to spread from otitis media or mastoiditis or from the hematogenous routetuberculosis, gonorrhea, scarlet fever, etc. Systemic etiology may include ankylosing spondylitis, rheumatoid arthritis, or psoriasis.

Classically, hemarthrosis following trauma is the pathogenic factor for bone formation in TMJ ankylosis [3]. Condylar trauma may lead to hemarthrosis due to injury to the periosteum and capsular ligament. When this intracapsular hematoma organizes, hypertrophic bone is formed from the disrupted periosteum or metaplasia of non-osteogenic connective tissue. This may lead to hypomobility, and bony ankylosis may eventually develop.

Yan et al. in 2014 [4] put forth the hypotheses of hypertrophic nonunion. He described the sagittal fracture of condyle along with displacement of the disc. Herein, trauma also occurs in the glenoid fossa, thereby establishing the microenvironment in the articular surfaces for bone healing.

Electronic Supplementary Material The online version of this chapter (https://doi.org/10.1007/978-981-15-1346-6_65) contains supplementary material, which is available to authorized users.

S. Anchlia $(\bowtie)$

Department of Oral and Maxillofacial Surgery, Government Dental College and Hospital, Ahmedabad, Gujarat, India
When the condylar trauma is recent, mouth opening will exert a dual effect on new bone formation. This is explained in Fig. 65.1. Restricted jaw movement is not a determinant but rather a promoting agent for ankylosis. Injuries to both the articular disc and the articular surfaces are prerequisites to TMJ ankylosis.

\subsection{Clinical Features (Fig. 65.2)}

In ankylosis, TMJ movements may be partially or completely restricted in opening, protrusion, and lateral excursions. Palpation of joint movements is better in fibrous than bony ankylosis. In children even in bony ankylosis, joint movements can be palpated because of stretching in the cranial sutures.

Ankylosis results in issues with mastication, digestion, speech, and oral hygiene. This may lead to caries, periodontitis, encumbered eruption of mandibular molars, crowding of teeth, and anterior open bite. Mandibular incisors often show supra-eruption and labial tipping as nature's compensation since the mandible is placed much posterior to the maxilla, thereby reducing lip competence. In severe cases, the lower lip may be trapped under the maxillary incisors.

As the vertical growth of the ramus is restricted, the lower face is significantly shortened. The digastric and mylohyoid muscles produce marked antegonial notching at the inferior part of the mandible just anterior to the insertion of the masseter and medial pterygoid. Failure of condylar growth impedes the forward and downward movement of mandible resulting in localized thickening of the bone at the angle due to subperiosteal apposition which accentuates the antegonion. The mandibular warping is caused due to this, as well as the obtuse angle between the inferior border of the mandible and the base of the skull [5]. The long-standing contractions of the masticatory muscles also give rise to elongation and thickening of the coronoid process, ramal shortening, and chin recession. 
Fig. 65.1 Dual effect of mouth opening on new bone formation in recent condylar trauma

\section{a}

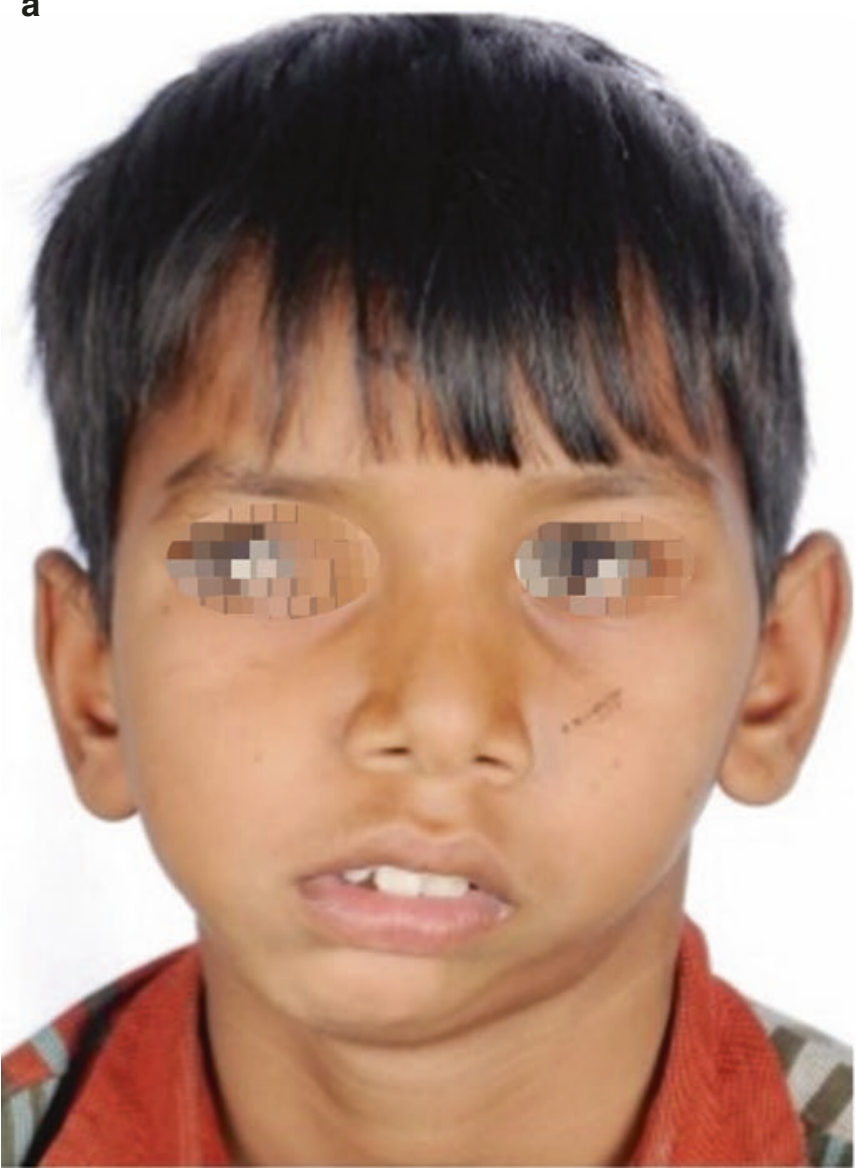

CAssociation of Oral and Maxillofacial Surgeons of India

Fig. 65.2 (a) Unilateral ankylosis of the right side, (b) bilateral ankylosis b

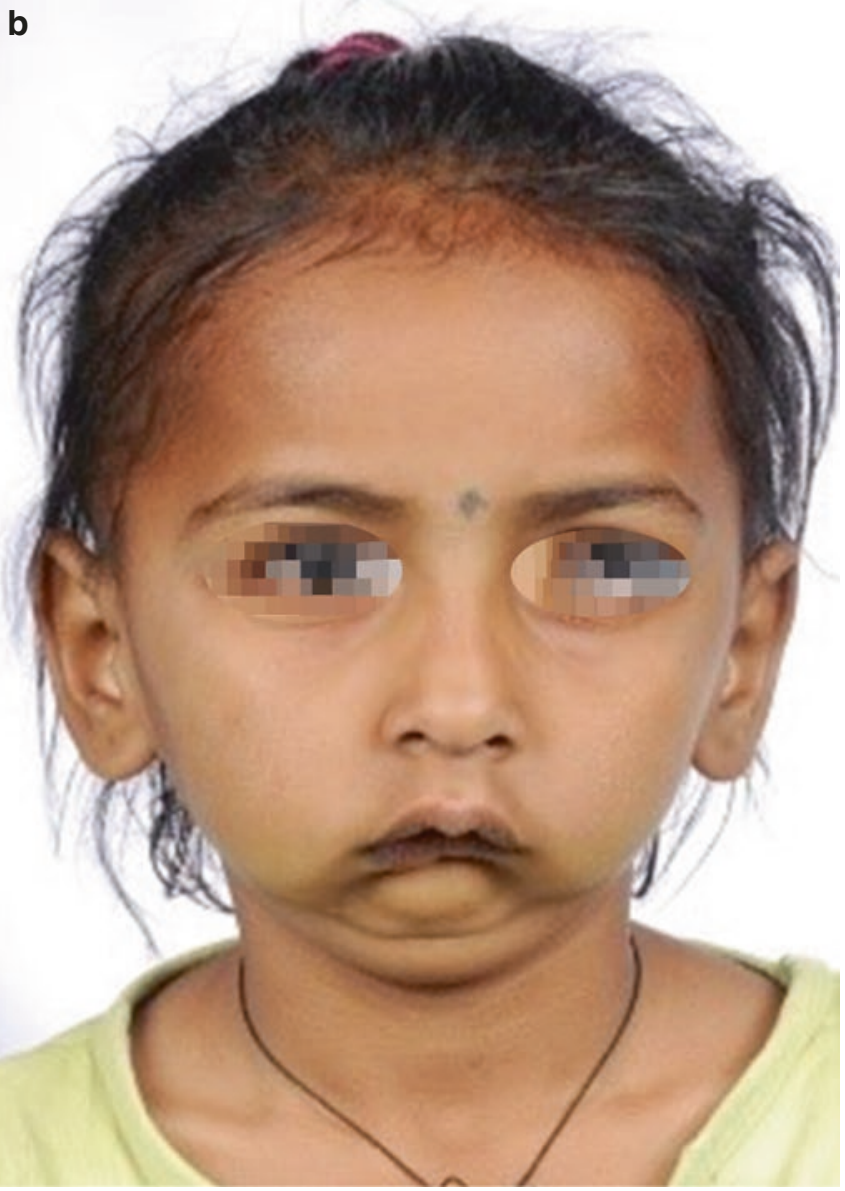


In unilateral cases, the chin deviates to the affected side due to restricted mandibular growth on the affected side and normal growth on the unaffected side. In growing patients, this may also restrict ipsilateral growth of the maxilla, although there is normal growth on the unaffected side. In some cases, the growth of the maxilla on the affected side may be normal but that on the unaffected side may be excess, due to uninhibited growth of the normal side. Both these phenomena result in a maxillary cant, though on opposite sides.

In bilateral cases, the mandible is visibly retruded, lacks a chin and the patient presents with an obtuse cervicomental angle. The posterior maxillary height is shortened, giving rise to steep occlusal planes. This convex facial profile or the bird face deformity is also described as the "Andy Gump deformity." Andy Gump was a cartoon character with a very insignificant chin, who first appeared in the Chicago Tribune in 1917, created by cartoonist Sidney Smith [6].

Worsened aesthetics of their appearance often becomes a reason for depressive disorders, psychological problems such as stubbornness, low self-esteem, and an inferiority complex.

The longer the duration of hypomobility, the more severe is the muscle atrophy and facial asymmetry. The prognosis for a favorable outcome with treatment is inversely related to the number of years of ankylosis.

\subsubsection{Importance of Obstructive Sleep Apnea-Hypopnea Syndrome (OSAHS)}

The presence of retruded mandible and micrognathia in patients with TMJ ankylosis creates narrowing of the pharyngeal airway space (PAS) with mechanical obstruction to respiration, more so in the supine position and during sleep. This process forms a complex syndrome of apneic episodes with significant reduction in the mean oxygen saturation levels and secondary cardiac and respiratory problems, known as OSAHS [7]. Therefore, for patients in whom the release of TMJ ankylosis is carried out without the advancement of the mandible for the correction of OSA, there is worsening of an already compromised airway. In such cases wide mouth opening during postoperative jaw exercises can lead to upper airway collapse-a sense of choking, bradycardia, and restlessness or apnea-like episodes, hence increasing chances of ankylosis [8].

\subsubsection{Radiographic Features of Bony Ankylosis (Fig. 65.3)}

According to Yan et al. [4], the deformed TMJ is characterized by an enlarged condyle, thickened temporal bone, excessive bone formation, and a radiolucent zone in the bony fusion area. No scattered calcified dots are found in the radiolucent zone, showing that ossification occurs only in the existing bones.

Bony fusion is located in the lateral part of the joint with decreased/absent bone marrow cavity and osteosclerosis. The medial non-bony fusion area shows the atrophic condylar head and rudimentary joint space. Here, the morphology of the bone marrow cavity and bone mineral density is similar to that of normal bone.

Wu et al. [9] demonstrated that in fibrous ankylosis, there is fibrous tissue intruding into the bone marrow of the condyle as well as degeneration of the condylar cartilage. In bony ankylosis, there occurs new bone formation on the rough ankylotic surface of the condyle and slight bone degeneration.

\subsection{Classifications}

Ankylosis has been classified by various authors according to its extent, site, development, heterotrophic bone formation, symptoms, and most recently, the extent of ankylosis guiding the treatment plan (Table 65.1).

\subsection{Preoperative Assessment}

\subsubsection{Investigations}

1. Detailed history, complete clinical examination, professional photographs, for documenting the:

(a) Age of onset of ankylosis

(b) Type, duration, and extent of ankylosis

(c) Type of joint injury or infection

(d) Maximal interincisal opening

(e) Dental characteristics and occlusion

(f) Type of facial deformity

(g) Previous surgery

2. Routine hemogram and pre-op major investigations

3. Radiological examinations for evaluation of extent of ankylotic mass, discrepancy of jaws, and treatment planning (Fig. 65.4)

(a) Orthopantomogram:

(i) Decreased joint space

(ii) Absence/presence of normal condylar and coronoid anatomy

(iii) Prominent antegonial notch

(iv) Markings for osteotomy cuts (for distraction)

(b) PA cephalogram:

(i) Chin deviation-Cg-ANS-Me (Crista Galli Anterior Nasal Spine - Menton)

(ii) Occlusal cant

(iii) Grummon's analysis 


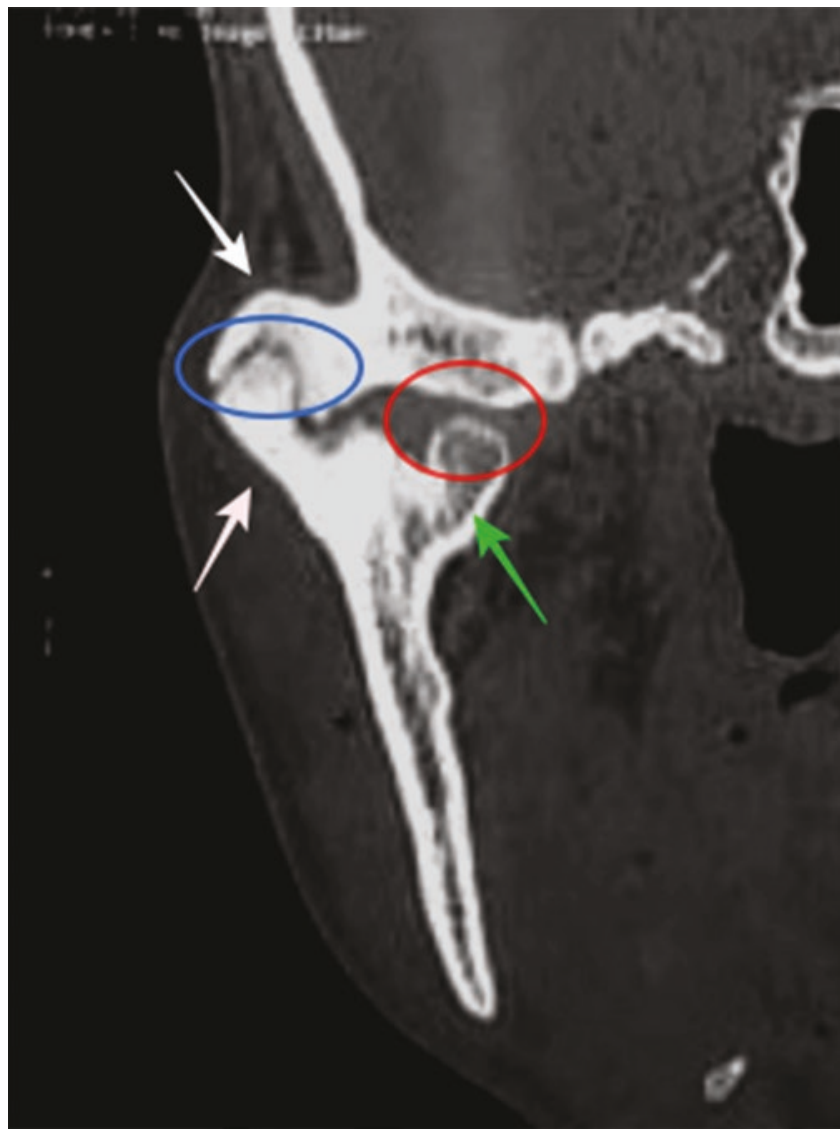

CAssociation of Oral and Maxillofacial Surgeons of India

Fig. 65.3 The computerized tomographic features of TMJ bony ankylosis. The blue circle refers to the bony fusion area located in the lateral part of the joint; in this area, a radiolucent zone can be observed. The red circle refers to the atrophic condylar head and rudimentary joint space located in the medial part of the joint. The green arrow indicates that bone mineral density and morphology of the bone marrow cavity in the non-bony fusion area were normal. The white arrows indicate excessive bone formation around the joint

(c) Lateral cephalogram:

(i) Ramal length: Ar-Go (Articulare-Gonion)

(ii) Corpus length: Go-Pog (Gonion-Pogonion)

(iii) Pharyngeal airway space (PAS)

(iv) $\mathrm{N}$ perpendicular to Pog (Nasion perpendicular to Pogonion)

(d) Facial CT scan:

(i) Three-dimensional anatomy of bony morphology

(ii) Any anatomical measurements as and when required, e.g., size of ankylotic mass, location of ligula, airway space volume, etc.

(e) CT Angiography may be required to assess the relationship of internal maxillary artery to the ankylotic mass. There are chances of the vessel being inside the bone, especially in re-ankylosis cases.

4. Assessment of OSA may be done using Epworth sleepiness scale, Pittsburgh sleep quality index, and polysomnography (PSG) for:
(a) Apneic-hypopneic index (AHI)
(b) Nocturnal desaturation episodes
(c) Average respiratory disturbance index
(d) Average lowest arterial oxygen saturation

5. 3D stereolithographic models printed, with the help of CT scan, may be used in treatment planning. Patientspecific surgical guides aid planning, precise control during operation, and thus more foreseeable treatment results.

\subsubsection{Airway Implications of TMJ Ankylosis}

\subsubsection{Assessment}

McNamara's airway analysis on lateral cephalogram helps to assess the PAS, CT provides volumetric assessment of the same, whereas PSG helps to assess the AHI. Moderate to severe AHI may require delayed extubation/tracheostomy.

\subsubsection{Types of Intubation According to Clinical Situations}

Blind nasal intubation was done historically, but it was traumatic, unpredictable, and unsafe. Tracheostomy is a traumatic experience, but nowadays, awake intubation is performed using midazolam and fentanyl [10]. Fiberopticassisted nasotracheal intubation has become the gold standard for such patients.

However, emergency tracheostomy during/after extubation may be required if:

(a) Bilateral ankylosis release is performed, but RCU reconstruction for ramal height rehabilitation has not been done.

(b) OSA is severe and release precedes mandibular advancement.

In these two situations, the mandible may fall back as there is no posterosuperior stop for the ramus and may impinge on the airway, causing further increase in OSA.

\subsection{Surgical Anatomy [11]}

\subsubsection{Nerve Anatomy (Figs. 46.6 and 65.5)}

\subsubsection{Facial Nerve}

The main trunk of the facial nerve exits from the skull at the stylomastoid foramen. Approximately $1.3 \mathrm{~cm}$ of the facial nerve is visible until it divides into temporofacial and cervicofacial branches.

According to Al-Kayat and Bramley (1979) [12], the distance from the lowest point of the bony external auditory canal (EAC) to this bifurcation measures $1.5-2.8 \mathrm{~cm}$ (mean $2.3 \mathrm{~cm}$ ). The postglenoid tubercle to the bifurcation measures $2.4-3.5 \mathrm{~cm}$ (mean $3.0 \mathrm{~cm}$ ). However, the point at which the upper trunk crosses the zygomatic arch is the most vari- 


\section{Table 65.1 Classifications}

\section{Topazian (1964)}

- Stage 1: Ankylotic bone limited to the condylar process

- Stage 2: Ankylotic bone extending to the sigmoid notch

- Stage 3: Ankylotic bone extending to the coronoid process

II. Sawhney's classification (1986)

- Type 1: Minimal bony fusion but extensive fibrous adhesions around the joint

- Type 2: Bony fusion at the outer edge of the articular surface but no fusion on medial area of the joint

- Type 3: Bridge of bone between the mandible and temporal bone

- Type 4: Joint is replaced by a mass of bone

\section{Turlington and Durr (1993)}

According to heterotopic bone formation within the ankylotic mass

- Grade 0: No bone islands visible

- Grade 1: Islands of bone visible within the soft tissue around the joint

- Grade 2: Periarticular bone formation

- Grade 3: Apparent bony ankylosis

Grades 1, 2, and 3 are further classified as symptomatic (S) and asymptomatic (A)

IV. Recent advances: Dongmei He and Colleagues (2011)

\section{Type of Ankylosis}

- Type A1: Fibrous ankylosis without bony fusion of joint

- Type A2: Bony fusion on lateral side of joint, residual condyle bigger than half of condylar head on medial side

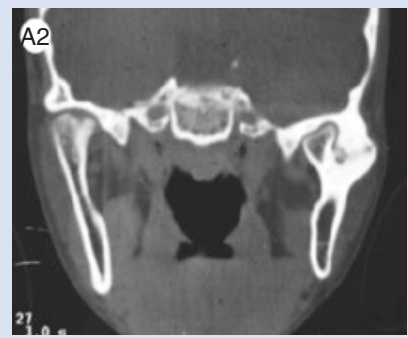

- Type A3: Similar to A2 but residual condylar fragment is smaller than half

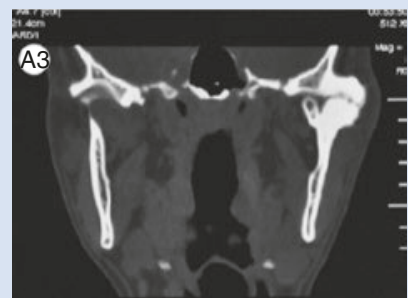

- Type A4: Ankylosis with complete bony fusion of joint

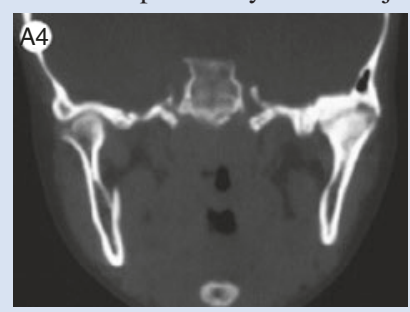

V. Recent advances: Yan and colleagues (2014)

Based on its development, ankylosis can be classified into three phases:

- Fibrous-chondral phase demonstrating fibrous tissue and chondrocytes occupied the joint gap

- Chondral-calcified cartilage phase manifesting abundant chondrocytes, cartilage matrix, and neo-formative endochondral ossification in the joint space

- Bone-cartilage phase showing compacted bone bridge in the lateral joint gap and cartilage in the medial joint gap

\section{Suggested Treatment Plan}

- Ankylosis release temporalis muscle flap, costochondral graft

- Lateral arthroplasty

- Resection of ankylotic mass, costochondral graft

- Resection of ankylotic mass, costochondral graft 
able in terms of distance. It may be anywhere from 8 to $35 \mathrm{~mm}$ anterior to the most anterior portion of the bony EAC (mean $2.0 \mathrm{~cm}$ ). If the superficial layer of the temporalis fascia and the periosteum over the arch is incised within $8 \mathrm{~mm}$, one can prevent injury to the upper trunk branches.

However, Miloro et al. [13] showed that the average distance anterior to bony EAC was $2.12 \mathrm{~cm}$. $(1.68-2.49 \mathrm{~cm})$. His was an MRI-based study as compared to cadaveric dissection-based work of Al-Kayat and Bramley.

The temporal branch of the facial nerve emerges from the parotid gland and crosses the zygoma under the temporoparietal fascia to innervate the frontalis muscle in the forehead.
Postsurgical palsy manifests as an inability to raise the eyebrow and ptosis of the brow. Damage to the zygomatic branch results in paralysis to the orbicularis oculi. Nerve damage may necessitate either tarsorrhaphy or gold weight implants into the upper eyelid for gravity-assisted closure. Transcutaneous nerve stimulation may be helpful in cases of neuropraxia.

\subsubsection{Trigeminal Nerve}

The auriculotemporal nerve courses from the medial side of the posterior neck of the condyle and turns superiorly, running over the zygomatic root of the temporal bone.
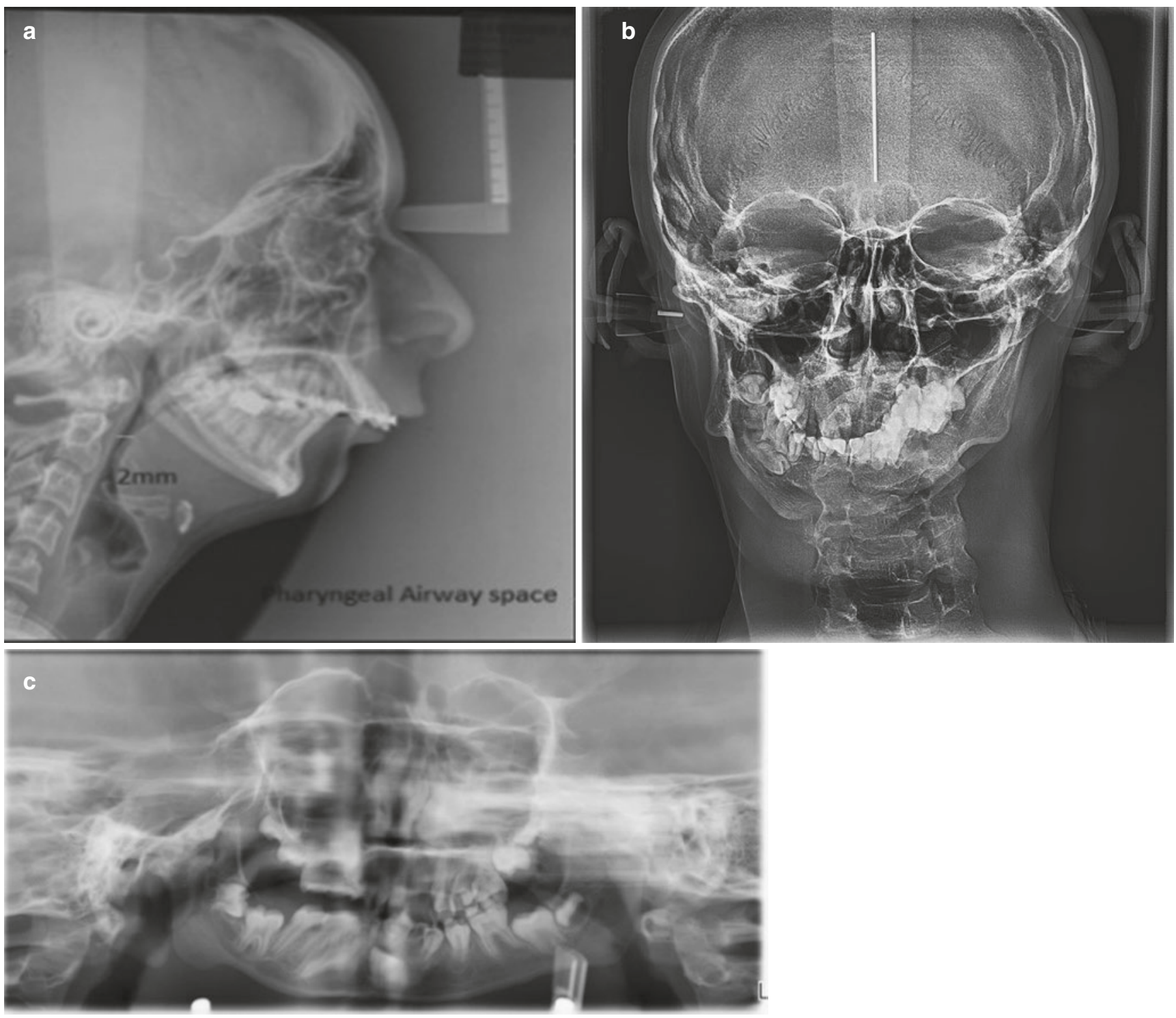

Fig. 65.4 (a) Lateral cephalogram, (b) PA cephalogram, (c) orthopantomogram, (d) facial CT scan, (e) facial cone beam CT, (f) volumetric assessment of pharyngeal airway space on CBCT 

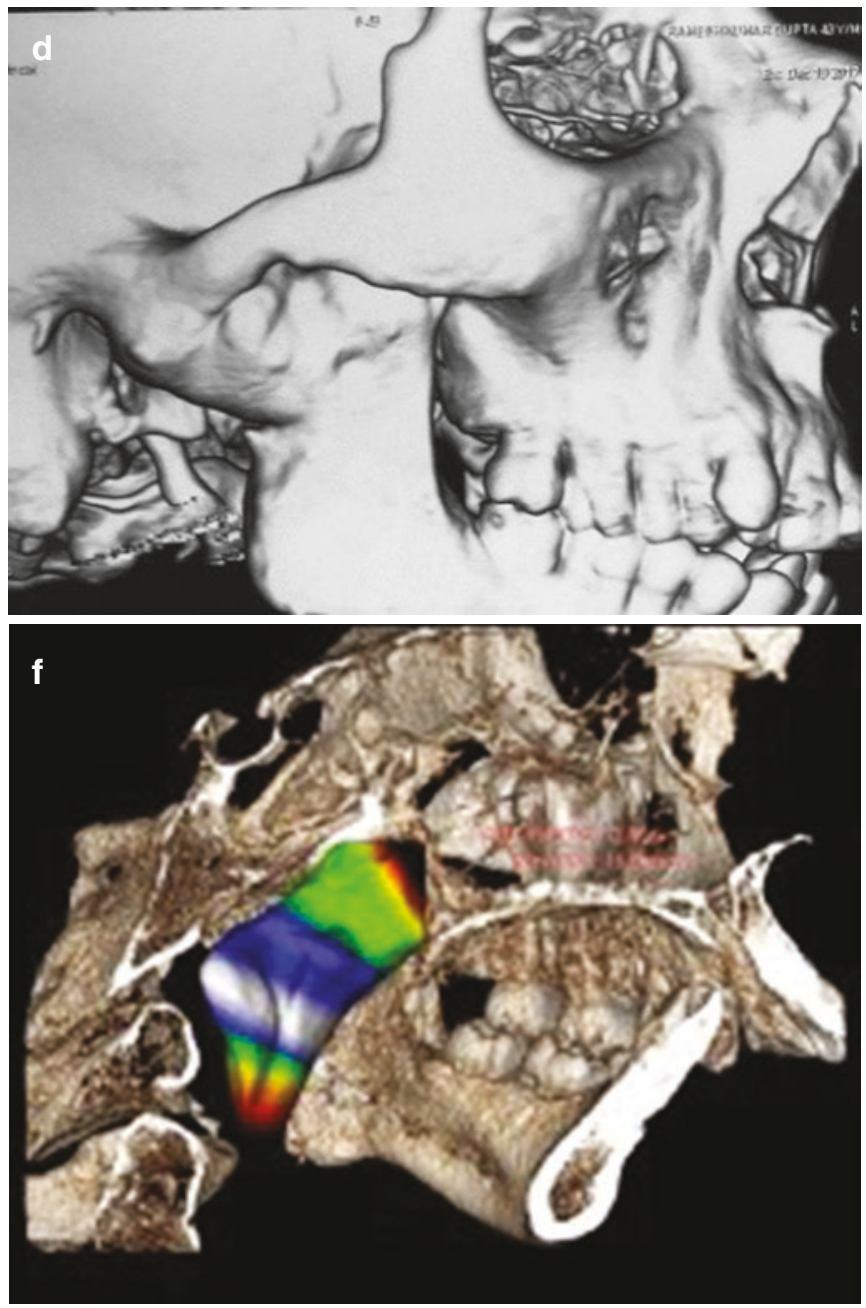

CAssociation of Oral and Maxillofacial Surgeons of India

Fig. 65.4 (continued)

Preauricular exposure of the TMJ area invariably injures the nerve. Damage is minimized by incision and dissection in close apposition to the cartilaginous portion of the external auditory meatus.

\subsubsection{Vascular Anatomy (Fig. 65.5)}

The superficial temporal artery and internal maxillary artery are the terminal branches of the external carotid artery. In the preauricular approach, the internal maxillary artery runs about $3 \mathrm{~mm}$ medial to the mid-sigmoid notch.

The most commonly injured artery during temporomandibular procedures is the middle meningeal branch of the internal maxillary artery. Pogrel [14] in a cadaver study of structures medial to the temporomandibular joint found the middle meningeal artery to be a mean of $31 \mathrm{~mm}(21-43 \mathrm{~mm})$ medial to the zygomatic arch and a mean of $2.4 \mathrm{~mm}(2-8 \mathrm{~mm})$ anterior from the height of the glenoid fossa.

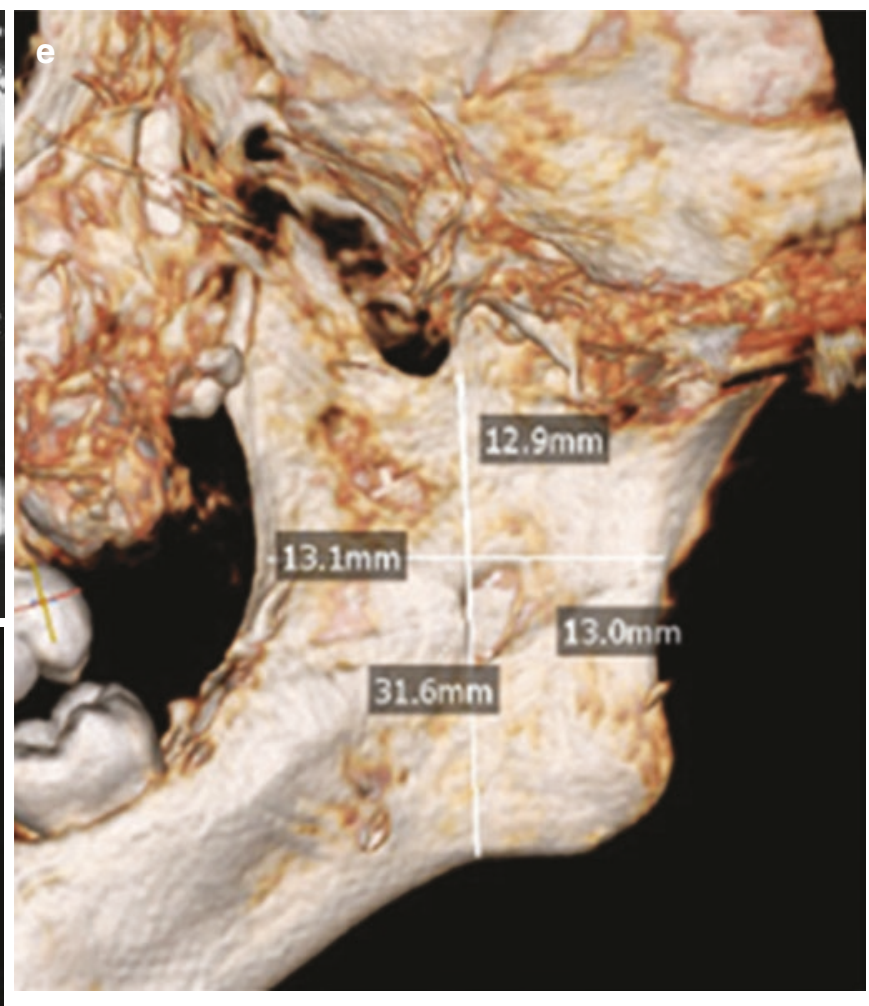

65.5.3 Incisions (Fig. 65.6) [5]

\subsubsection{Dingman's Preauricular Approach (Refer video on Pre auricular approach in Chap. 53 and Fig. 53.17)}

It is the most commonly used incision with many variations. Classically, the incision begins along the course of the helix, just in front of the tragus till the attachment of the ear lobule. Going through the skin and superficial fascia, about $2 \mathrm{~cm}$ above the zygomatic arch, an oblique incision is made through the superficial layer of temporal fascia. Just above the arch, the periosteum of the zygomatic arch is incised and turned forward as one flap with the outer layer of the temporal fascia, superficial fascia containing nerves, and skin. The ankylotic mass now stands exposed.

\subsubsection{Blair (1914)}

It is like the Dingman's incision in preauricular fold, but unlike the former it bends in the region of the zygomatic arch 

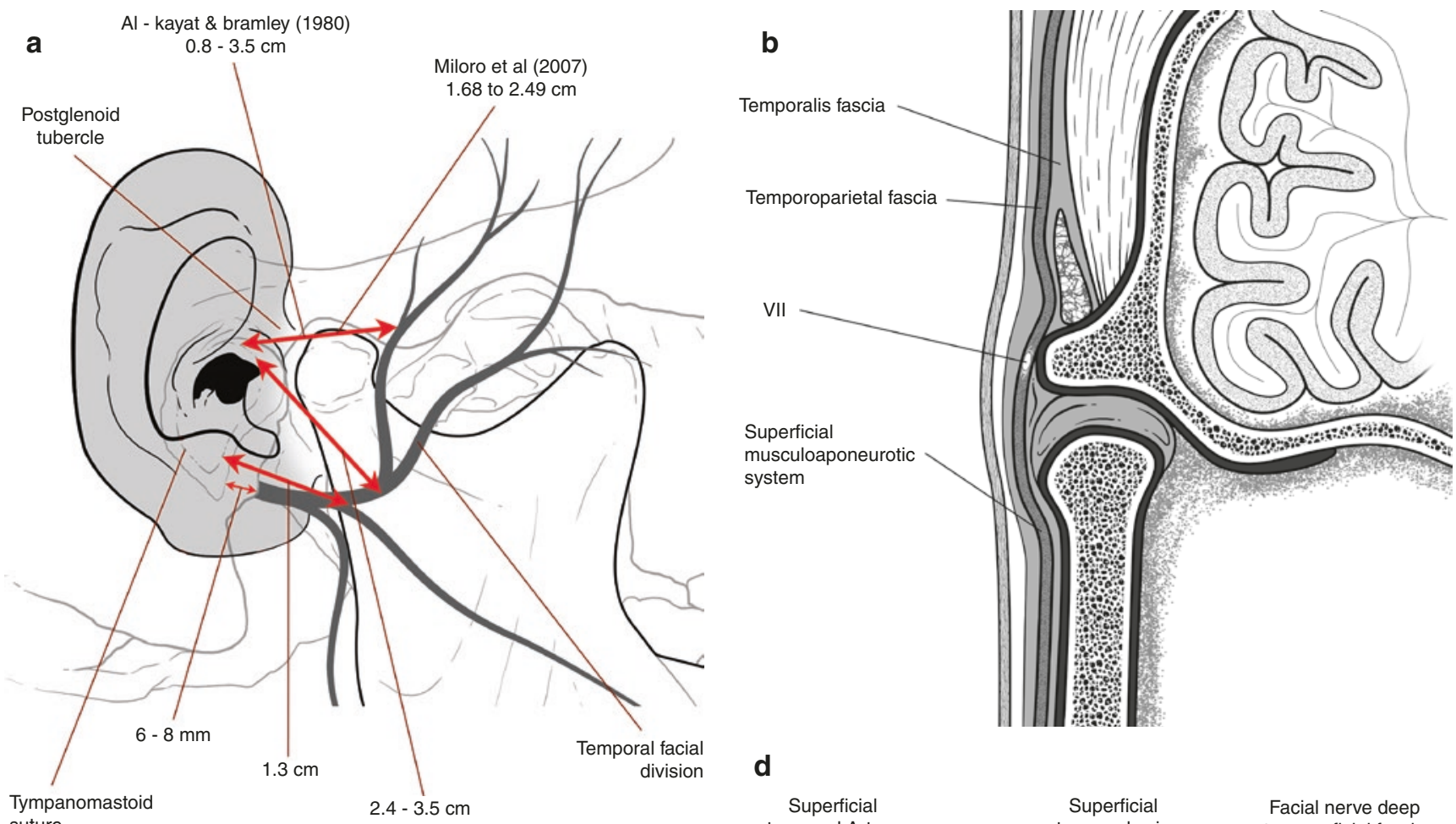
suture

d
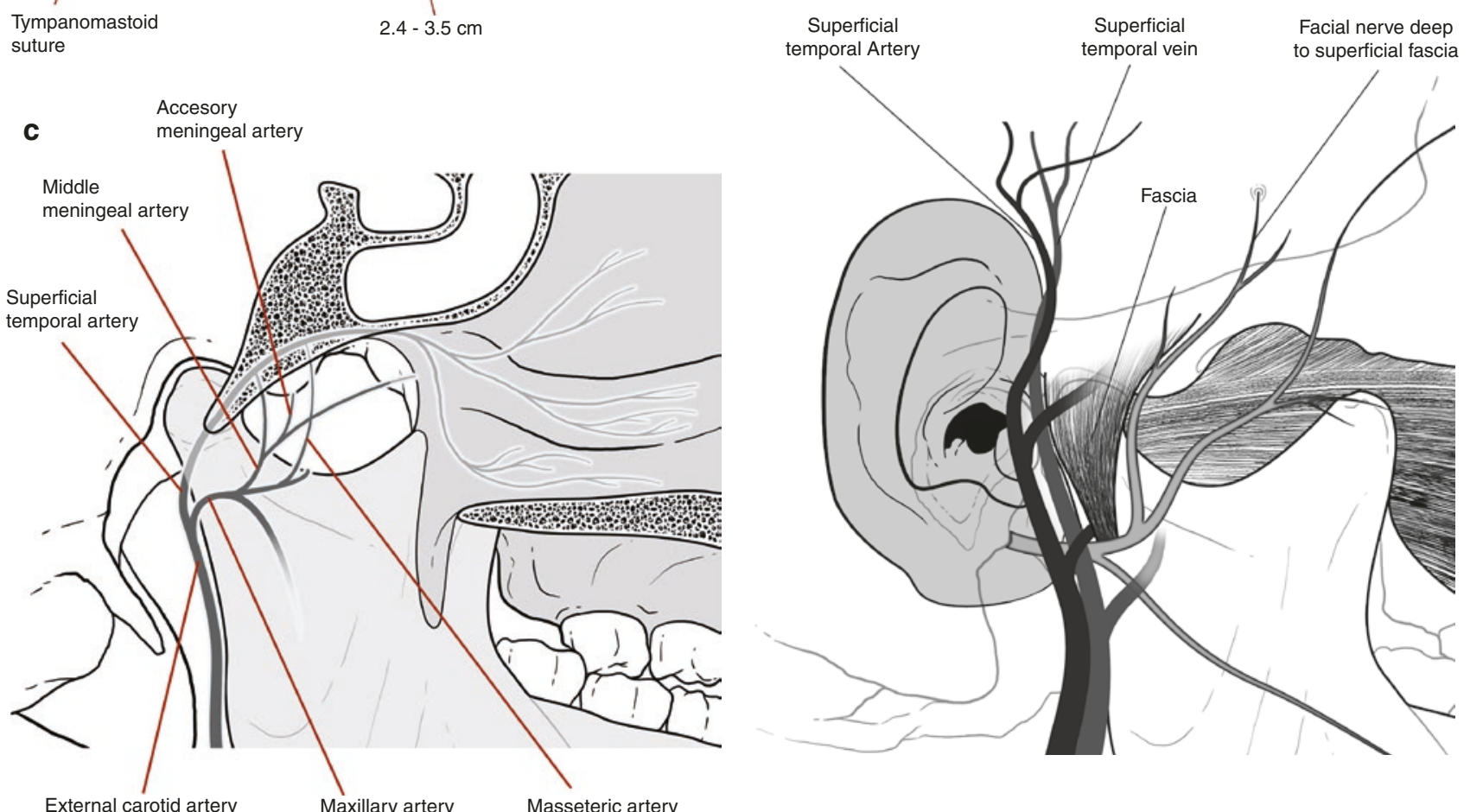

(C)Association of Oral and Maxillofacial Surgeons of India

Fig. 65.5 (a) Surgical landmarks for identifying the location of main trunk of the facial nerve and the temporal-facial division during temporomandibular arthroplastic dissection. (b) Coronal diagram of the fascial layers and facial nerve at the level of the temporoparietal fascia. (c) View from medial aspect of the mandible. Note proximity of middle

meningeal, external maxillary, and masseteric arteries. Care should be taken to protect these structures at the level of the condylar neck and sigmoid notch during osteotomies. (d) Relative position of the superficial temporal artery and vein and the temporal branch of the facial nerve 

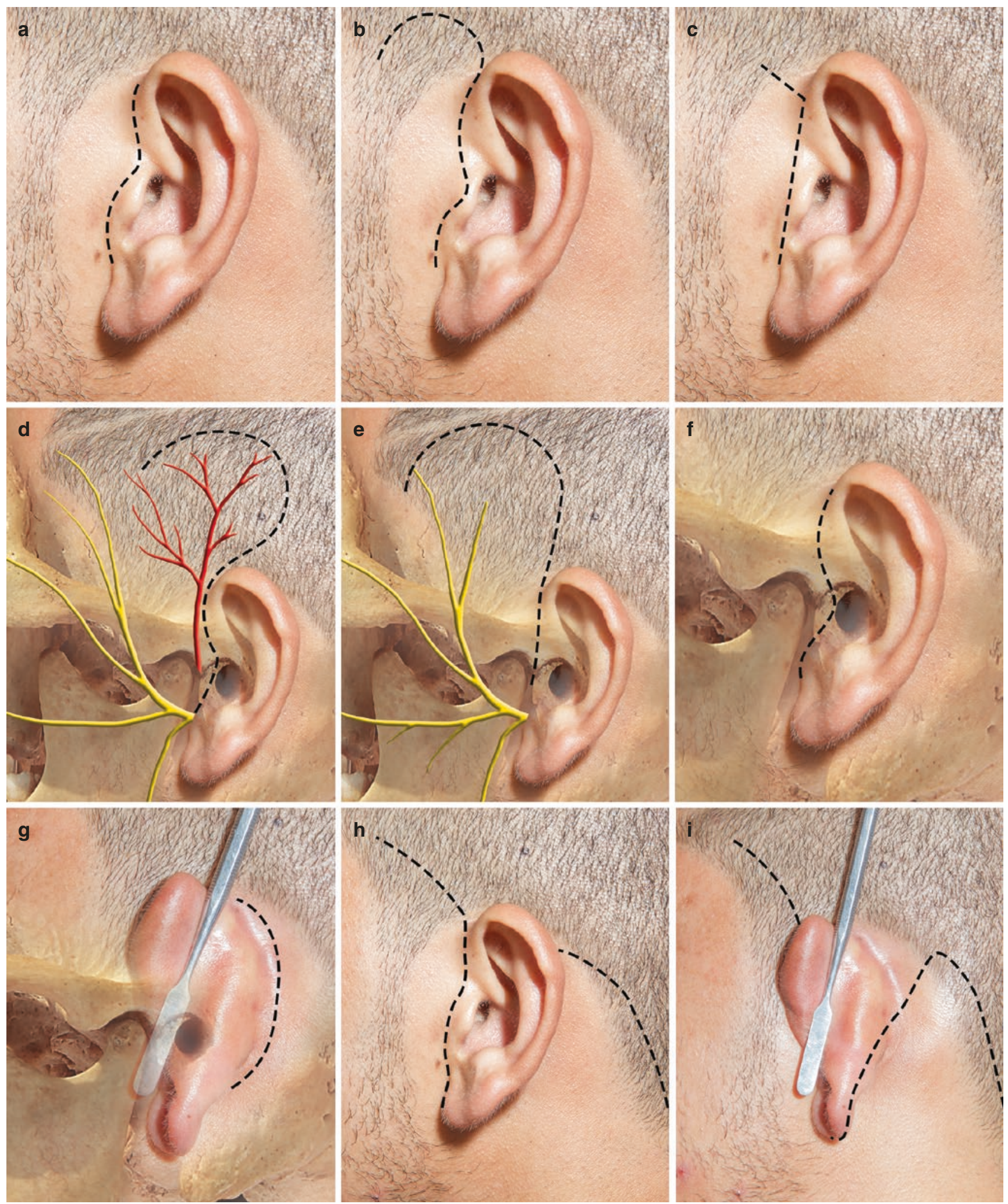

CAssociation of Oral and Maxillofacial Surgeons of India

Fig. 65.6 (a) Dingman, (b) Blair's inverted hockey stick, (c) Thoma's, (d) Al-Kayat and Bramley. (e) Popowich's modification, (f) endaural, (g) postauricular, (h) rhytidectomy, (i) retro-auricular limb of rhytidectomy incision 
like a hockey stick. The disadvantages include an unsightly scar and possible damage to the frontal branch of the facial nerve.

\subsubsection{Thoma (1945)}

The vertical limb is in the preauricular fold but angulated at $45^{\circ}$ in the hairline near the bifurcation of the superficial temporal vessels.

\subsubsection{Al-Kayat and Bramley (1979) (refer video on Al Kayat Bramley approach in Chap. 53)}

This reverse question mark cosmetically acceptable incision gives excellent access to the TMJ without causing any damage to important anatomical structures. It starts superiorly through the scalp in the temporal region and extends to the inferior tragus. The superficial layer of the temporalis fascia is identified and incised at the root of the arch at $45^{\circ}$ anterosuperiorly to avoid branches of the facial nerve. A vertical incision may be used if the temporalis fascia or muscle is to be used.

\subsubsection{Popowich's Modification of Al-Kayat and Bramley [5]}

This approach to the zygomatic arch and joint gives excellent visibility with safety. It is longer and wider than the conventional and question mark shaped and begins about a pinna's length away from the ear, anteroposterior just within the hair line, curves backward and downward well posterior to the main branches of the temporal vessels, till it meets the upper attachment of the ear.

\subsubsection{Endaural [10]}

This was first described by Rongetti in 1954, with the incision carried in the external ear. The advantages are good aesthetics and excellent access to the TMJ. However, the disadvantages include perichondritis, infection, paresthesia of the pinna, and deformity of the ear.

\subsubsection{Coronal Extension of Preauricular Incision}

The anterosuperior extension over the scalp of the preauricular incision gives a more aesthetic scar.

\subsubsection{Rhytidectomy [10]}

The endaural incision is extended in a curvilinear fashion around the mastoid tip, with an $\mathrm{S}$-shaped extension ending in a submandibular incision. This allows access to the entire posterior border of the mandible and allows for identification of the main trunk of the facial nerve.

\subsubsection{Postauricular}

First described by Alexander [15] in 1975, its biggest advantage is that it avoids facial nerve injury and salivary fistula. However, possible complications include stenosis of external auditory canal, infection, and paresthesia of the pinna.

\subsection{Treatment Protocol}

Kaban [2] and colleagues described an approach for the treatment of TMJ ankylosis to minimize the incidence of re-ankylosis and produce satisfactory movement of the joint. Their study in 1990 became a landmark management protocol for the management of TMJ ankylosis (Table 65.2).

Yet again in 2009, Kaban [16] considered the potential effect of time and growth (i.e., the fourth dimension) on the outcome of TMJ ankylosis in children and presented another protocol (Table 65.2).

\subsubsection{Surgical Options for Ankylosis Release} (Fig. 65.7a-c)

History Ankylosis release surgeries, i.e., various ways of separation of the ramus from the skull have undergone great evolution during the nineteenth century. Esmarch [17] osteotomized the angle to release TMJ ankylosis in 1851. The first interpositional material was the pterygomasseteric sling, also developed by Esmarch. Condylectomy was first advocated by Humphrey in 1854. Abbe performed the first gap arthroplasty in 1880. But it was Topazian in the 1960s $[18,19]$ who signified the importance of interpositional grafts for reducing re-ankylosis.

\subsubsection{Gap Arthroplasty}

The conventional surgical treatment of TMJ ankylosis by gap arthroplasty includes complete resection of the ankylotic

Table 65.2 Kaban's protocols for TMJ ankylosis

1. Kaban's protocol for management of temporomandibular joint ankylosis (1990) [2]

(a) Aggressive resection of the ankylotic segment

(b) Ipsilateral coronoidectomy

(c) Contralateral coronoidectomy when necessary

(d) Lining the joint with temporalis fascia or cartilage

(e) Reconstruction of the ramus with a costochondral graft

(f) Rigid fixation of the graft

(g) Early mobilization and aggressive physiotherapy

2. Kaban's modified protocol for management of TMJ ankylosis in children (2009) [16]

(a) Aggressive excision of fibrous and/or bony mass

(b) Coronoidectomy on affected side

(c) Coronoidectomy on opposite side if steps 1 and 2 do not result in MIO of $35 \mathrm{~mm}$ or to point of dislocation of opposite side

(d) Lining of joint with temporalis fascia or the native disk, if it can be salvaged

(e) Reconstruction of RCU with either DO or CCG and rigid fixation

(f) Early mobilization of jaw; if DO used to reconstruct RCU, mobilize day of surgery; if CCG used, early mobilization with minimal intermaxillary fixation (not $>10$ days)

(g) Aggressive physiotherapy 

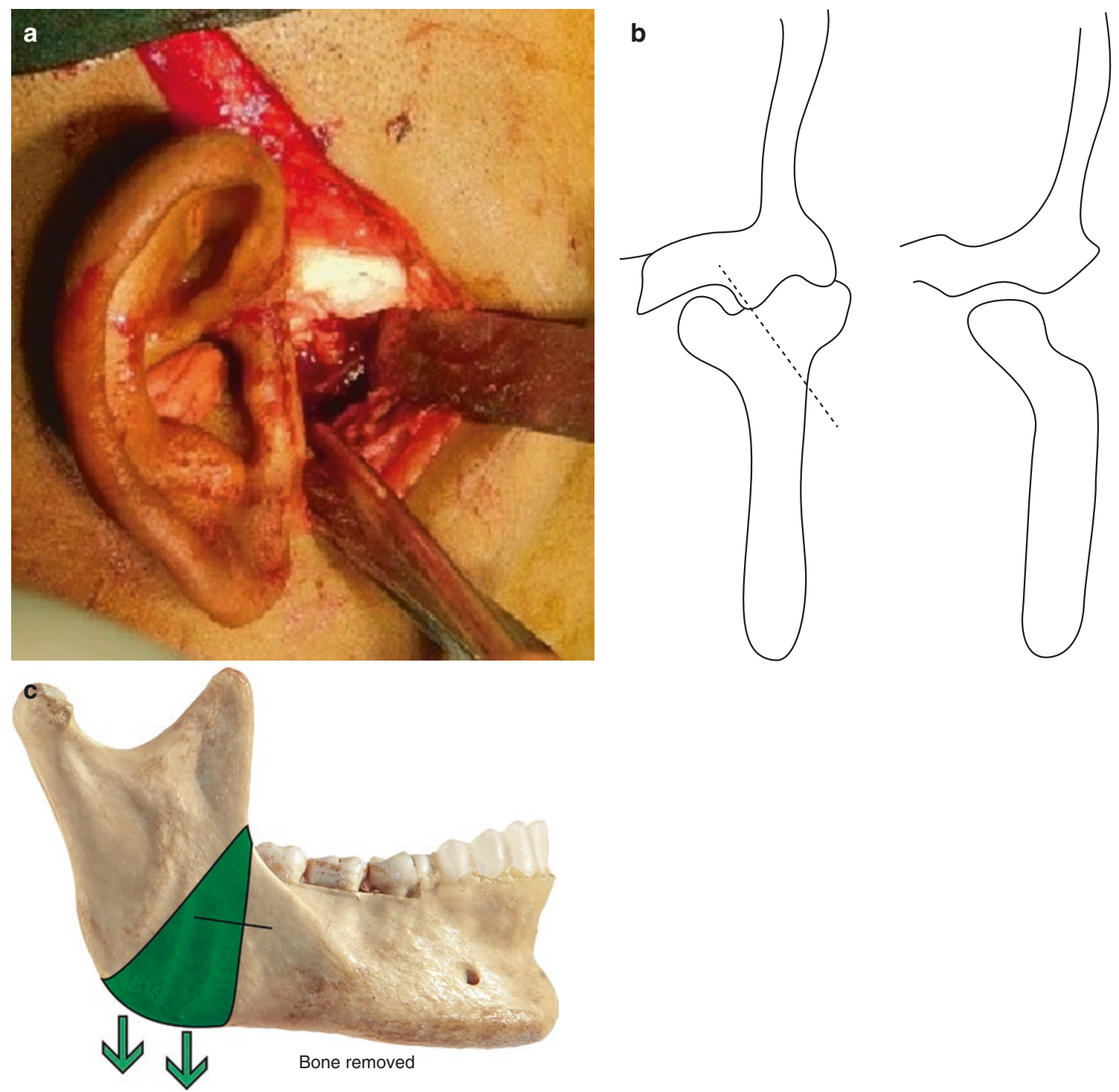

CAssociation of Oral and Maxillofacial Surgeons of India

Fig. 65.7 (a) Gap arthroplasty, (b) Lateral arthroplasty (LAP), (c) Esmarch's procedure

mass with no soft tissue as an interposition between two cut ends. When a soft tissue is placed between the cut ends, it is known as interpositional arthroplasty. Gap arthroplasty is a relatively simple procedure requiring less operative time; however, it may cause ramal shortening, malocclusion, OSA, and, most distressingly, re-ankylosis. But it may still be used in certain adult patients, where the removal of a small ankylotic mass would not decrease the ramal height significantly.

Patients are operated under general anesthesia using a preauricular approach. After the ankylotic mass is exposed, the upper cut is marked, and the lower cut is placed $1.5 \mathrm{~cm}$ below it. In cases where normal architecture of the joint is maintained, the lower osteotomy cut is placed from the anterior to the posterior border of ramus just below the sigmoid notch. In cases where normal architecture is not clear, a cut is placed $1.5 \mathrm{~cm}$ below the lower border of zygomatic arch. Then the upper cut is completed, and the ankylotic mass is removed. The osteotomy is directed at $45^{\circ}$ to prevent fracture of middle cranial fossa. The venous plexus if encountered medially needs only be packed to control bleeding.

A condylar retractor is kept below the ankylotic mass to prevent injury to the internal maxillary artery. Bone is cut initially using a bur/saw/piezoelectric unit through two thirds of its thickness. The remaining one thirds may be removed with an osteotome. A Piezoelectric unit if used, may cut bone till its very end. In case of a larger mass, it may also be removed in pieces. The ipsilateral coronoid process is always resected. Care is always taken to stop the application of Heister's mouth gag intraoperatively just before the opposite condyle dislocates. 
In some patients with longstanding ankylosis, the coronoid process of the opposite site becomes hyperplastic. There may also occur fibrosis of the masseter, which would restrict jaw movements further. Thus, not only would the contralateral coronoidectomy be required, but the masseter attachment would also need to be completely dissected off the zygomatic arch. A coronoidotomy may not be adequate, as it may re form and attach again to the temporalis and subsequently cause decrease in mouth opening. An intact disc may not require another soft tissue interpositional graft but may itself be mobilized and repositioned into the new glenoid fossa.

R. F. Elgazzar [20] reviewed 101 patients (109 joints) and, on follow-up, reported that in comparison with other techniques of ankylosis release, gap arthroplasty had the least amount of mouth opening. Interpositional arthroplasty with temporalis gives better results in comparison.

Surgical navigation systems are useful in TMJ ankylosis surgery as they improve both the safety and accuracy of the surgery. The distance from the top of the glenoid fossa to the middle cranial fossa and that from the posterior margin of the fossa to the anterior border of the bony external auditory canal can be monitored via the navigation system. A safety distance of at least $3 \mathrm{~mm}$ from the middle cranial fossa and bony external auditory canal should be maintained to avoid injury. It reduces the risks of bleeding and CSF leak [21].

\subsubsection{Esmarch's Procedure}

This is of historical significance only. Esmarch [22] made a small incision posterior to the angle of the mandible and dissected the masseter and medial pterygoid muscles off the bone. A $2.5 \mathrm{~cm}$ wide wedge of bone, at the angle of the mandible with apex at upper border and base at the posteroinferior border, was removed. The muscles were then sutured back.

The disadvantage is that since the osteotomy is in the ramus, no ramal osteotomy or distraction can be done for correction of facial deformity. Hence, it is no longer used for ankylosis release.

\subsubsection{Lateral Arthroplasty (LAP)}

Nitzan hypothesized that in Sawhney's [3] Type II TMJ ankylosis or He's Type A2 [23], if the integrity and location of the displaced condyle and disc can be determined (despite morphological and positional alterations), both could be preserved to fulfill their roles in mandibular growth and function. Only the lateral part of the ankylosed mass was resected. Eight young patients were kept under observation for a period ranging from 6 to 60 months after surgery. She found significant improvement in facial symmetry and thereby concluded that the residual condyle retained its own growth potential and helped maintain normal mandibular movement function and occlusion [24].
Yang and Dongmei He evaluated long-term results after LAP in nine growing children and concluded that the residual condyle continues to grow after LAP. This then decreases the amount of further facial asymmetry [25].

\subsubsection{Arthroplasty with Ramus-Condyle Unit (RCU) Reconstruction (Details Covered Under "Hard Tissue Interposition")}

Interpositional arthroplasty with RCU reconstruction is the procedure of choice in recent times. The procedure consists of:

(i) Removal of the ankylotic mass.

(ii) Reconstruction of the RCU may be considered from amongst any of the following in costochondral graft, coronoid process graft, distraction osteogenesis (DO), total alloplastic joint replacement.

Early postoperative exercises and appropriate physiotherapy are essential to prevent re-ankylosis, adhesions, and soft tissue contracture and to regain normal function of the muscles. Dynamic mouth opening exercise initiated in the immediate postop period by achieving pain control using local infiltration of bupivacaine. Dynamic mouth exercises would have to be continued for at least 6 months after surgery.

\subsection{Soft Tissue Interpositional Materials (Video 65.1)}

\subsubsection{Temporalis Myofascial Flap} (Fig. 65.8d, e)

Historically, it has been the most commonly used material for interpositional arthroplasty as it is simple in technique and in the vicinity of the original area of surgery. An inferiorly based pedicled composite (fascia and muscle), temporalis myofascial flap is raised supraperiosteally which is finger shaped and about $5 \times 2 \mathrm{~cm}$. The flap is taken either over/ under the zygomatic arch and sutured medially and posteriorly. However, its potential disadvantages include an extended incision, temporal hollowing, increased chances of facial nerve injury, inability to be used again in cases of reankylosis, and myofascial pain secondary to muscle flap compression.

Albert and Merril described the use of a posterior temporalis myofascial flap to prevent a visible anterior flap hollowing [26]. Pogrel and Kaban used an axial patterned temporalis myofascial flap, based on a branch of the middle temporal artery [27]. 


\subsubsection{Dermal Fat Graft (Fig. 65.8a, b, c)}

The abdominal dermal fat graft is harvested from the suprapubic area used an elliptical incision of around $7 \times 3 \mathrm{~cm}$. The fat is then de-epithelized to procure a volume of 6-20 $\mathrm{ml}$. About $20-30 \%$ excess is taken than the actual requirement. The graft is folded onto itself with the dermis surfaces apposed.

It can be procured quickly and easily with a hidden scar. It is easy to sculpt into the gap after ankylosis release.
Minimal heterotopic calcification is the advantage of dermal fat graft. However, since this is a non-vascularized graft, it may be prone to resorption.

Dimitroulis presented his study on dermal fat grafts. After a mean follow-up of 41.5 months, only 1 out of the 13 joints reported recurrence of ankylosis [28]. Thangavelu presented a review of eight ankylosed joints treated using full-thickness skin subcutaneous fat grafts and concluded that they were safe and effective [29].

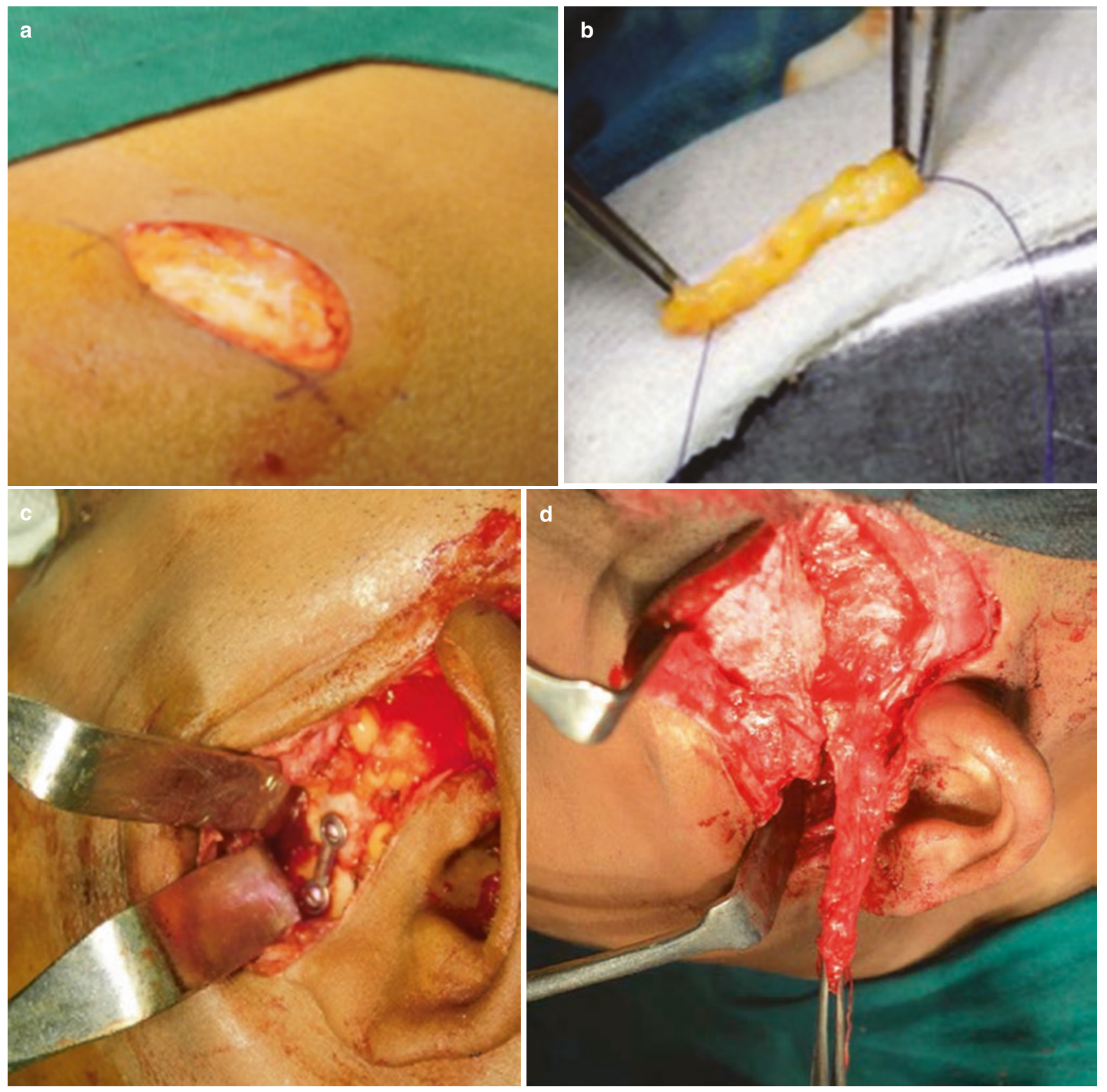

Fig. 65.8 (a) Abdominal fat graft donor site and (b) harvested graft, (c) abdominal fat graft in place, (d) harvested temporalis flap, (e) temporalis flap in place, (f) maneuver for harvesting buccal fat pad, (g) harvested buccal fat pad 

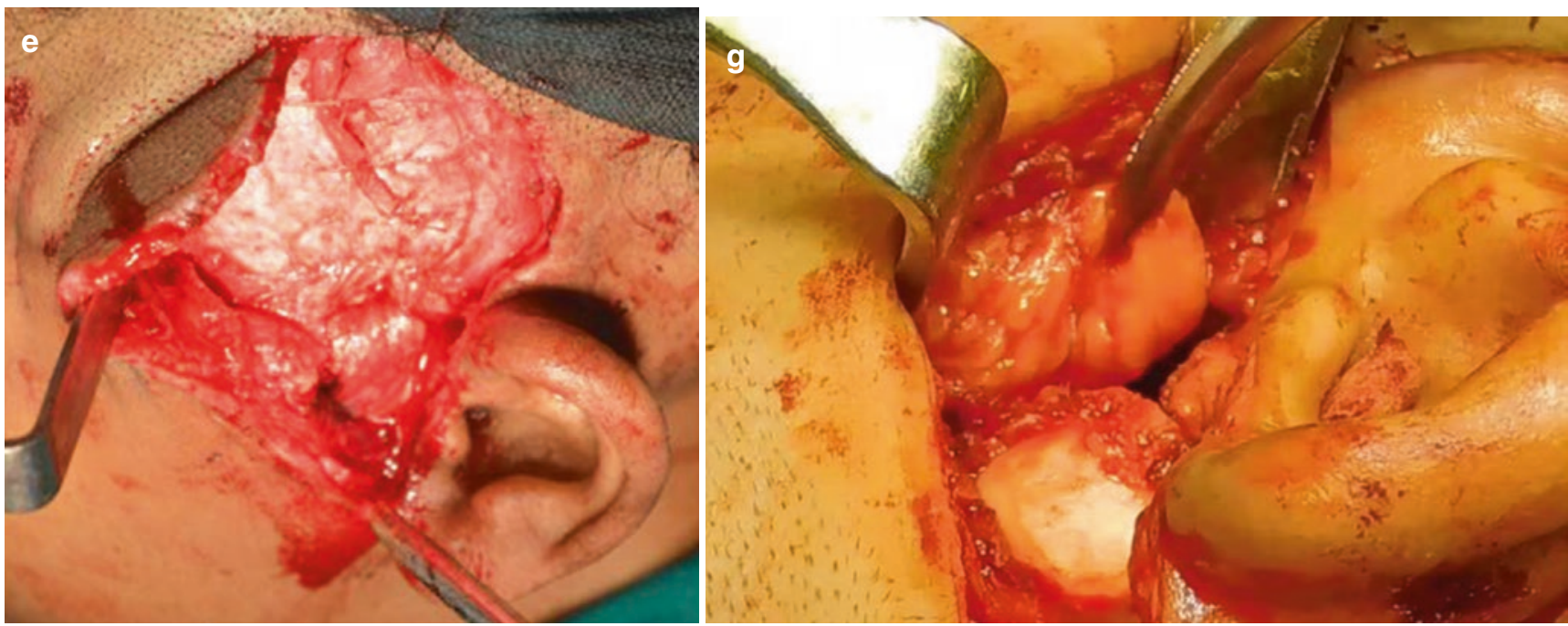

f

LATERAL

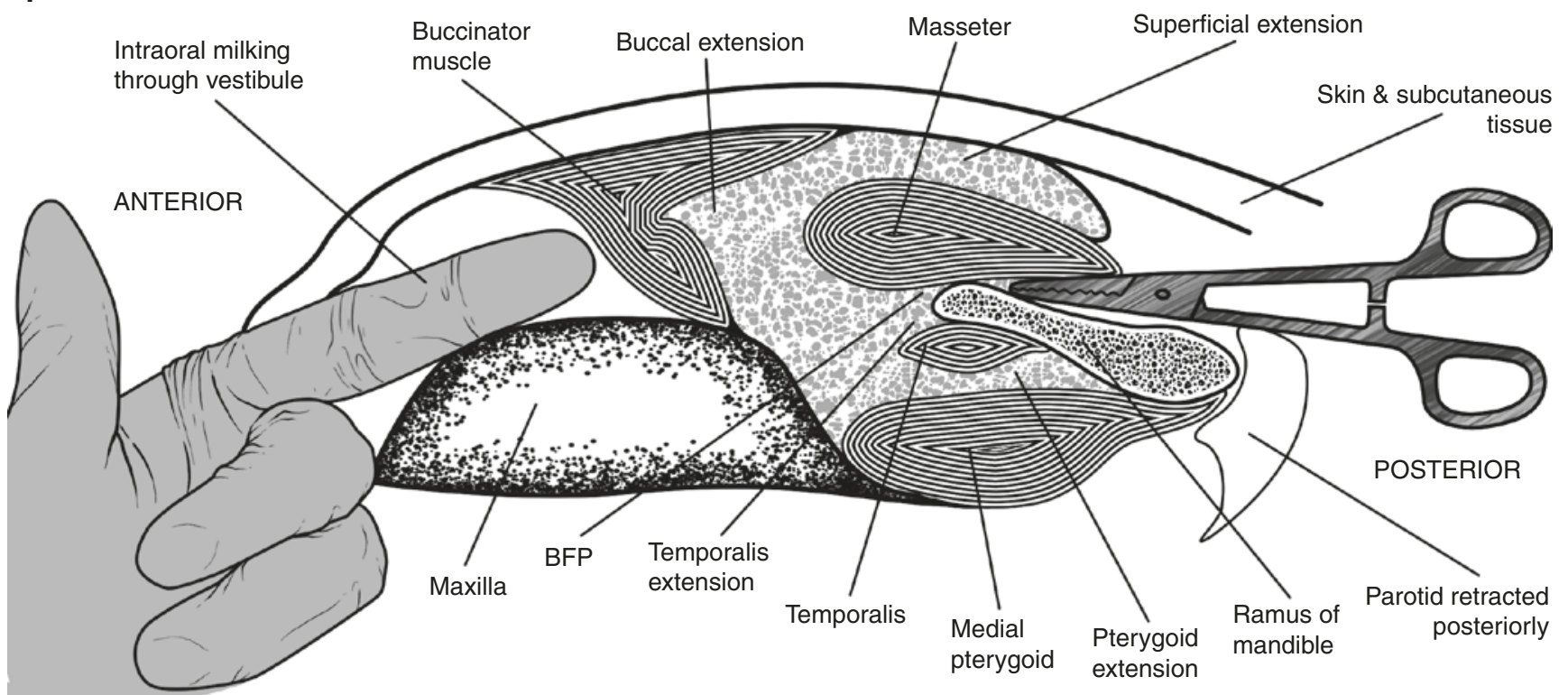

MEDIAL

CAssociation of Oral and Maxillofacial Surgeons of India

Fig. 65.8 (continued)

\subsubsection{Buccal Fat Pad Graft (BFP) (Fig. 65.8f, g)}

After the coronoid process is removed, dissection maybe performed along the anterior border of ramus. The main part and temporal extension of buccal fat pad are in close approximation to the coronoid process and tendon of the temporalis. Thus, incising through the periosteum and fascial envelope of BFP at the anterior most edge of the coronoid stump/superior most stump of anterior border of ramus exposes the yellowish buccal pad of fat.

Further blunt dissection is performed to herniate the BFP into the defect with a little teasing and simultaneous milking of maxillary buccal sulcus intraorally near second and third molar region. The main advantage is that its vascularity would maintain the volume of fat for a longer duration with minimal resorption.
Gaba et al. prospectively assessed the fate of BFP following interpositional arthroplasty in 23 joints. In 18 cases, BFP was viable even after 1 year [30]. Refer Chap. 24 for detailed anatomy of the Buccal fat pad.

\subsubsection{Amniotic Membrane}

The amniotic membrane, being a natural biologic entity, prevents inflammation, reduces scarring, and promotes healing and re-epithelialization. It also has pluripotent, non-antigenic properties. Akhter M. et al. used gamma radiation sterilized and freeze-dried amniotic membranes in 13 patients of TMJ ankylosis and showed that it prevented recurrence of ankylosis [31]. 


\subsubsection{Auricular Cartilage}

Parko in 1973 first published the use of aural cartilage to replace the meniscus. The biggest advantage of auricular cartilage graft is that it can be harvested from the same surgical field, remains viable and inert and takes the shape of the glenoid fossa.

However, there are studies that have reported adhesions between the auricular cartilage graft and the condyle, leading to re-ankylosis.

\subsection{Hard Tissue Interpositional Materials (RCU Reconstruction)}

\subsubsection{Rationale for RCU Reconstruction}

The goal of RCU reconstruction in TMJ ankylosis includes restoration of ramal height. This not only avoids occlusal discrepancies and deviation on mouth opening in unilateral cases but also anterior open bite in bilateral ankylosis. It also provides enough bone stock for future jaw corrective surgeries. The RCU may not contribute as much to facial aesthetics but impacts function greatly. In cases where RCU has not been reconstructed, the jaw deviates laterally when opening the mouth. When closing the mouth, tangential rather than vertical striking occurs on the occlusal surfaces, making mastication difficult.

Most importantly, in bilateral TMJ ankylosis after gap arthroplasty without RCU reconstruction, there are increased chances of OSA because the ramus falls back due to lack of posterosuperior stop. Also, it may be possible that restoring normal jaw movement and a symmetric mandible will allow future soft and hard tissue development. This would probably lead to decrease in facial asymmetry by giving as near normal anatomy as possible by restoration of growth spurts (Moss's functional matrix theory).

\subsubsection{Options, Techniques, Advantages, and Disadvantages}

\subsubsection{Ankylotic Mass (Fig. 65.9a)}

The ankylotic mass is recontoured and utilized to reconstruct the RCU and thereby restore the mandibular ramal height. All neocondyles after RCU reconstruction are fixed into position with miniplates/screws in the new glenoid fossa just $2 \mathrm{~mm}$ short of the soft tissue interpositional material after placing the patient into intermaxillary fixation (IMF).

R. Gunaseelan excised the ankylotic mass, recontoured and reimplanted it, and used it successfully for three cases of condylar reconstruction [32]. However, only if the ankylotic mass is resected as a whole (which poses greater risk to mid- dle meningeal artery) can it be recontoured in an adequate size to reconstruct the RCU, hence the difficulty in routine use.

\subsubsection{Coronoid Process Graft (Fig. 65.9b)}

Khadka and $\mathrm{Hu}$ first reconstructed RCU using the coronoid process [33]. Reshaped, the coronoid process provides good bone of adequate quality and quantity to reconstruct the RCU. Its membranous origin leads to lesser resorption. Also, a secondary donor site is not utilized. However, as the coronoid is a nonvascular graft, there does exist a very real possibility of its resorption.

Gagan Mehta and Shadab Mohammad conducted a study on 20 cases and reported that there did occur resorption of the coronoid in most of the cases. However, that did not change mandibular function and occlusion [34].

Another method of using the coronoid process is to use it as RCU with its attachment to the temporalis kept intact. Most of the attachment may be stripped of, and only the anterior part of temporalis may be kept attached to the tip of the coronoid. It may cause less resorption as it would be a pedicled graft. Yiming Liu studied 48 patients and compared free vs. pedicled coronoid process grafts and proved that pedicled grafts showed less resorption on prolonged followup [35].

\subsubsection{Costochondral Graft (CCG) (Fig. 65.9c)}

Poswillo popularized the procedure for using CCG for TMJ ankylosis in 1987. In unilateral cases, CCG may be taken from the fifth rib using a submammary incision. If required on both the sides, alternate ribs, i.e., fifth and seventh, are used [36].

CCG has various benefits including its growth potential, its biological compatibility and the fact that it can remodel to form a new condyle. However, donor site morbidity, resorption or excessive growth on the treated side, and inability to catch up with the speed of growth on the normal side are its potential demerits. Perrott and Kaban [16] described two types of overgrowth-(a) linear overgrowth causing an

\section{Box 65.1 Technical Tip}

To maximize the chances of survival rate of CCG, the following techniques may be followed: meticulous dissection of periosteum and perichondrium, harvesting alternate ribs to prevent pain and pleural tear, retaining intact periosteum and perichondrium at costochondral junction, sectioning the chondral before the osseous part to reduce fracture at the costochondral junction, and only 2-3 $\mathrm{mm}$ chondral portion to prevent overgrowth. 
a
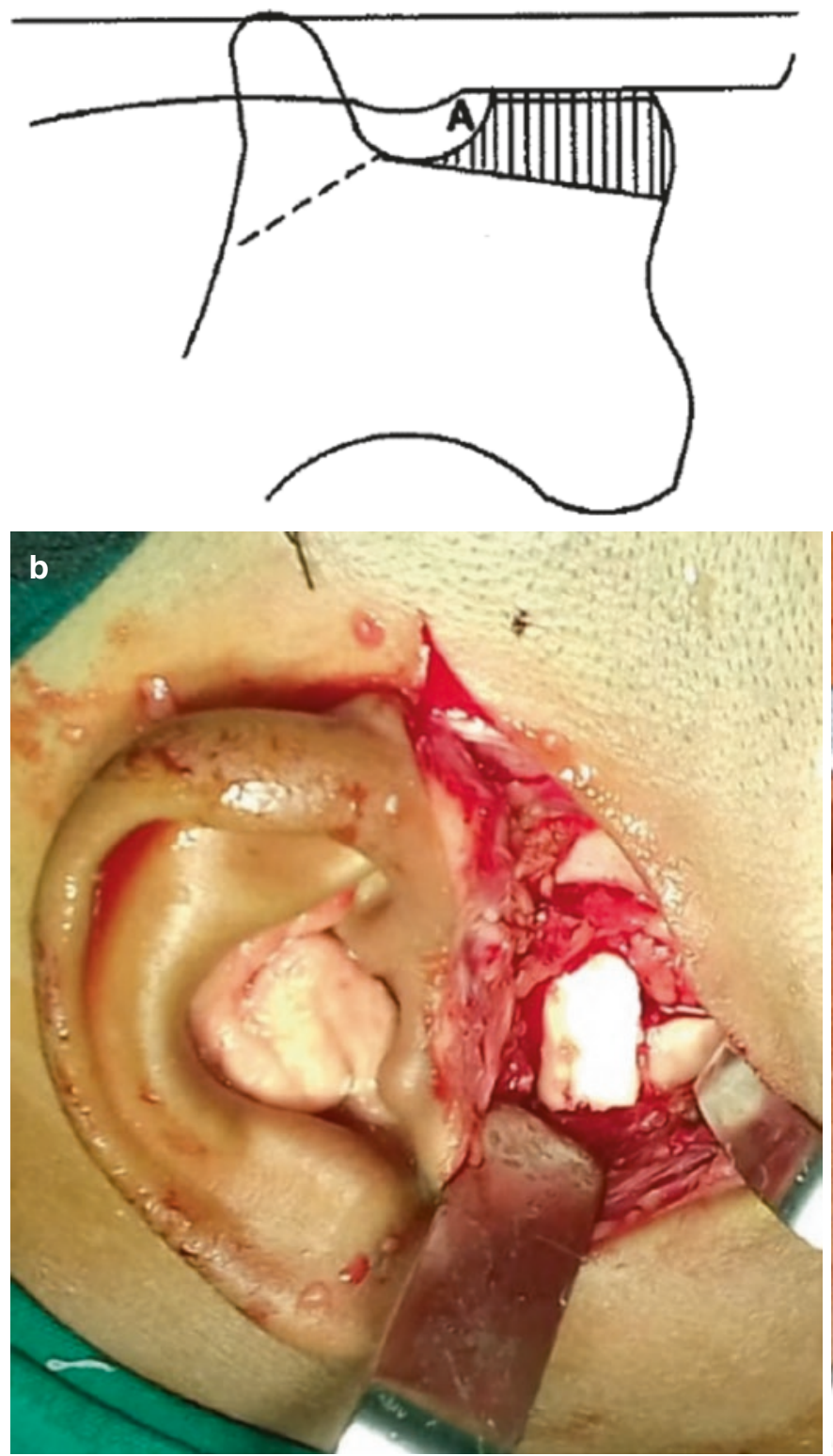

CAssociation of Oral and Maxillofacial Surgeons of India

Fig. 65.9 (a) Ankylotic mass, (b) coronoid graft, (c) costocondral graft

asymmetric or bilateral prognathism and (b) tumor-like overgrowth causing re-ankylosis.

There are various controversies regarding which ribs and how much cartilaginous cap should be taken, how to fix, whether post-op IMF is required or not, and resorption vs. hypertrophy of the graft. Jean Salash reported that out of 72 cases, excessive growth occurred in 54\% and equal growth occurred in $38 \%$ [37].

Hence, CCG may be used for RCU reconstruction in children less than 8 years of age with facial asymme-
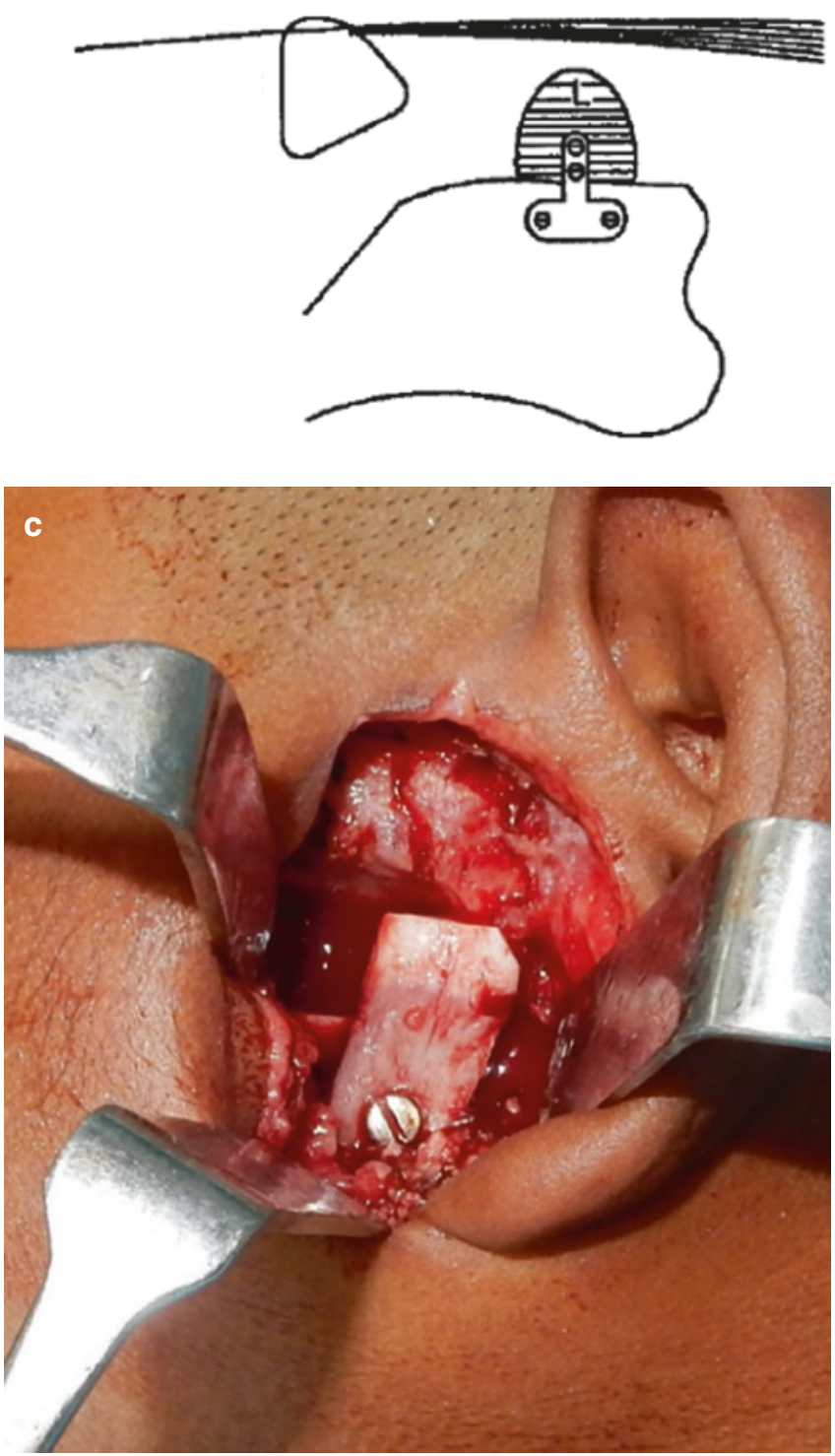

try. The justification is that it may help in growth. However, if it overgrows, the overgrowth may be treated like condylar hypertrophy, and the superior 2-3 mm may be cut off.

Children less than 8 years but without facial asymmetry would probably not need a growth impetus, in which cases, a coronoid graft would be sufficient. In children more than 8 years of age, vertical ramus osteotomies may be used to recreate the RCU. 


\subsubsection{Ramus Osteotomy Pedicled Grafts}

(Fig. 65.10a, b)

Y. Liu [38] used the method of total (Vertical ramus osteotomy - VRO) and partial (L- ramus osteotomy_LRO) sliding osteotomy on the posterior border of the ramus for reconstructing the mandibular condyle in TMJ ankylosis.

The chances of bony resorption and graft failure of the newly reconstructed RCU are greatly reduced as this segment is a pedicled graft with attachment to the medial pterygoid muscle and periosteum and is not a free graft. Hence the height of the ramus is maintained. This also leads to adequate bone stock for further corrective surgeries if and when so required.

For the VRO, the cut is made in a vertical direction starting from the sigmoid notch to the inferior border of the mandible just lateral to the lingula and parallel to the posterior border of the ramus. While retaining an adequate amount of medial pterygoid, the proximal segment is pushed upward to recreate the RCU and plated in the new position. A small triangular chunk of bone just anterior to the osteotomy cut is resected to reshape the lower border and mandibular angle.

For the LRO, an L-shaped cut is made from sigmoid notch parallel to the posterior border of the mandibular ramus, just lateral to the lingula, till $10 \mathrm{~mm}$ above the angle of the mandible. The osteotomized segment is shifted upward and fixed with a mini plate creating a gap between the osteotomized segment and remaining mandibular angle. The coronoid process on the affected side is resected, recontoured, and fixed in that gap with another mini plate. The upper part of the ramus is then reshaped like a condyle.

LRO and VRO differ in the residual height of the ramus left after ramal osteotomy. In LRO it is retained, and in VRO it decreases due to the removal of the step at the inferior bor-
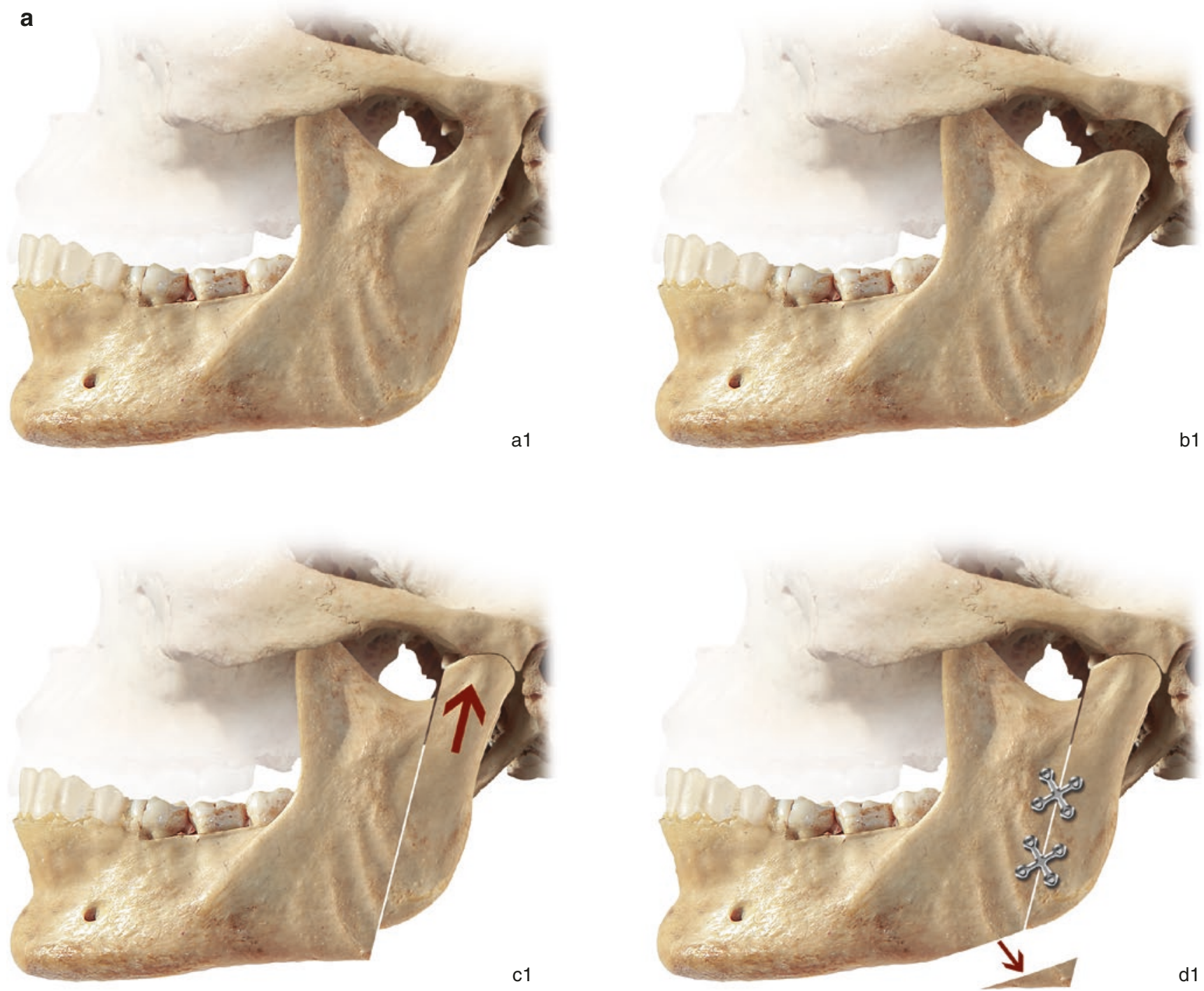

Fig.65.10 (a) V Ramus osteotomy, (b) L Ramus osteotomy [38] 

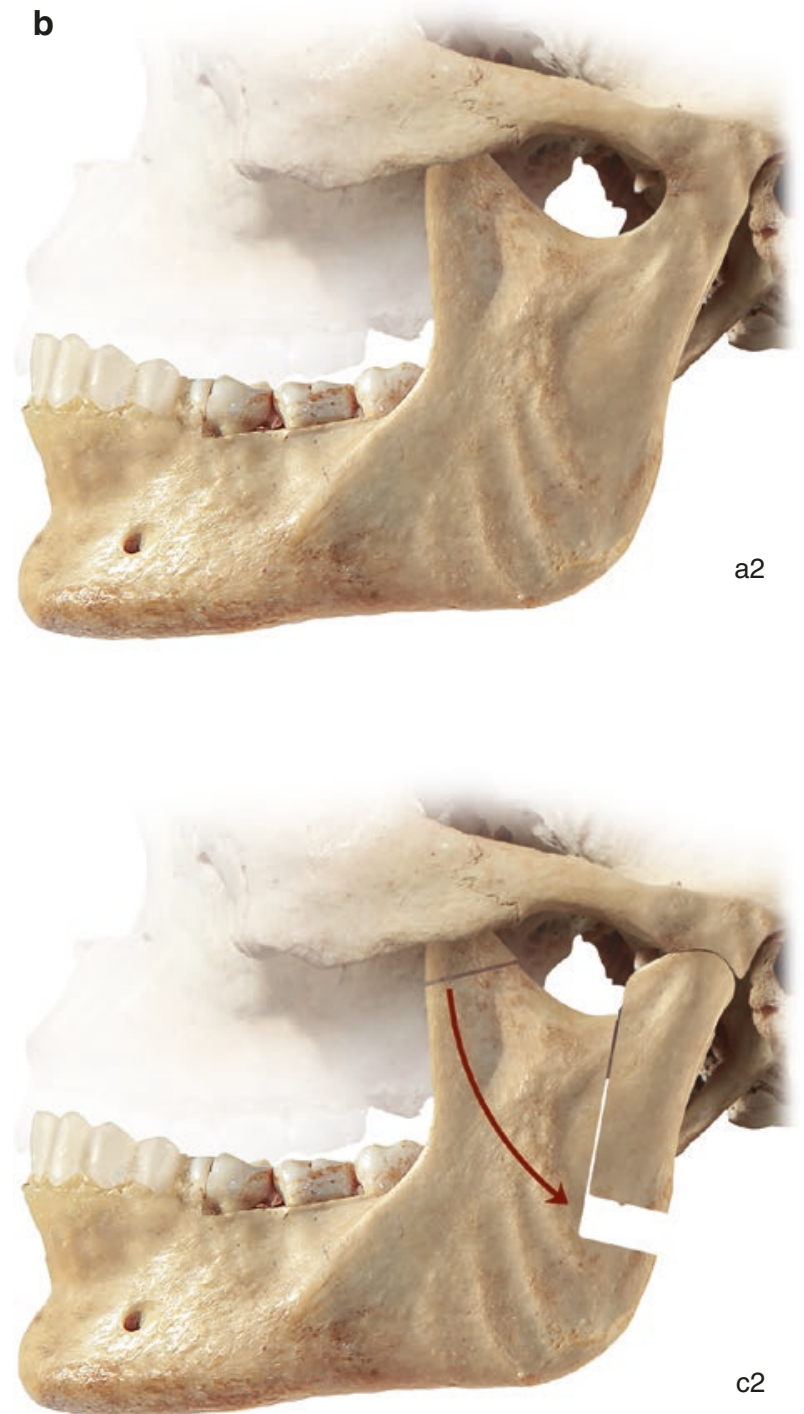

(CAssociation of Oral and Maxillofacial Surgeons of India

Fig. 65.10 (continued)

der. Thus, VRO may be performed in cases where a pronounced antegonial notch is present.

However, these osteotomies cannot be performed in patients less than 8 years because as observed on CBCT, the amount of bone behind the lingula is generally not enough to osteotomize the posterior border of the ramus.

\subsubsection{Transport Distraction Osteogenesis} (Fig. 65.11a, b) (Refer Chap. 87)

In multiple operated patients, scar tissue forms with each surgery, which may affect angiogenesis due to limited diffusion. Thus, free autogenous tissue grafts, such as costochondral and coronoid grafts often fail in such cases. Hence, transport distraction osteogenesis is considered as an option

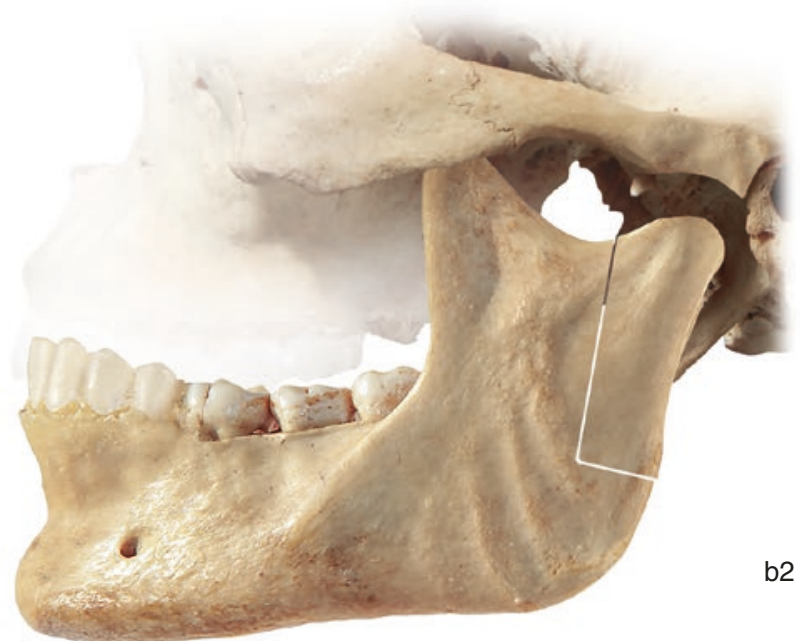

b2

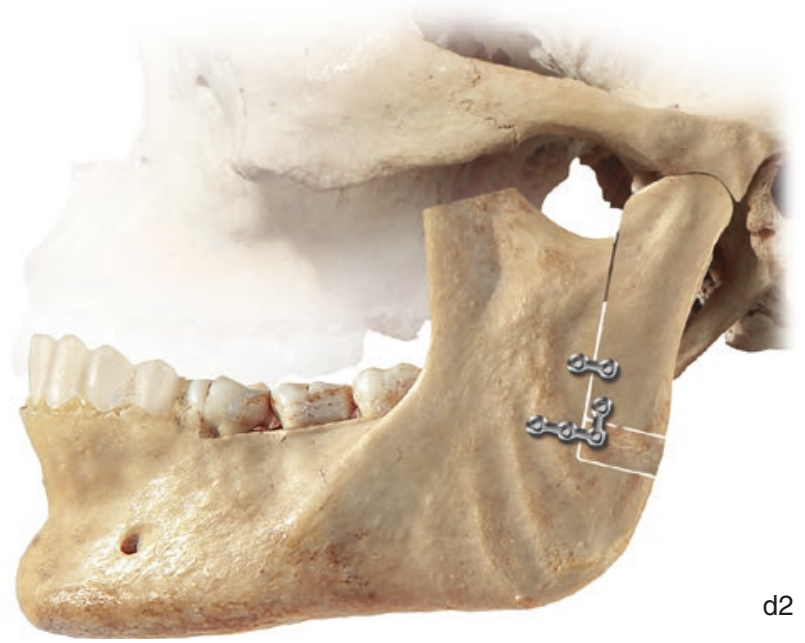

for RCU reconstruction, especially in re-ankylosis cases in children. The same L osteotomy cut may be used to fix the distractor.

When RCU reconstruction is performed by transport distraction, it forms a fibrous/pseudo-disc by compressing the connective tissue between the transport segment and the glenoid fossa. This disk decreases the chances of reankylosis [39]. Sharma et al. in 2019 carried out neocondyle distraction in five patients and reported adequate mouth opening and functional movements in all patients. None of them reported re-ankylosis [40]. But a long-term follow-up study found that the height of these condyles was unstable. Also, these mandibles exhibited some degree of asymmetry [41]. 
a
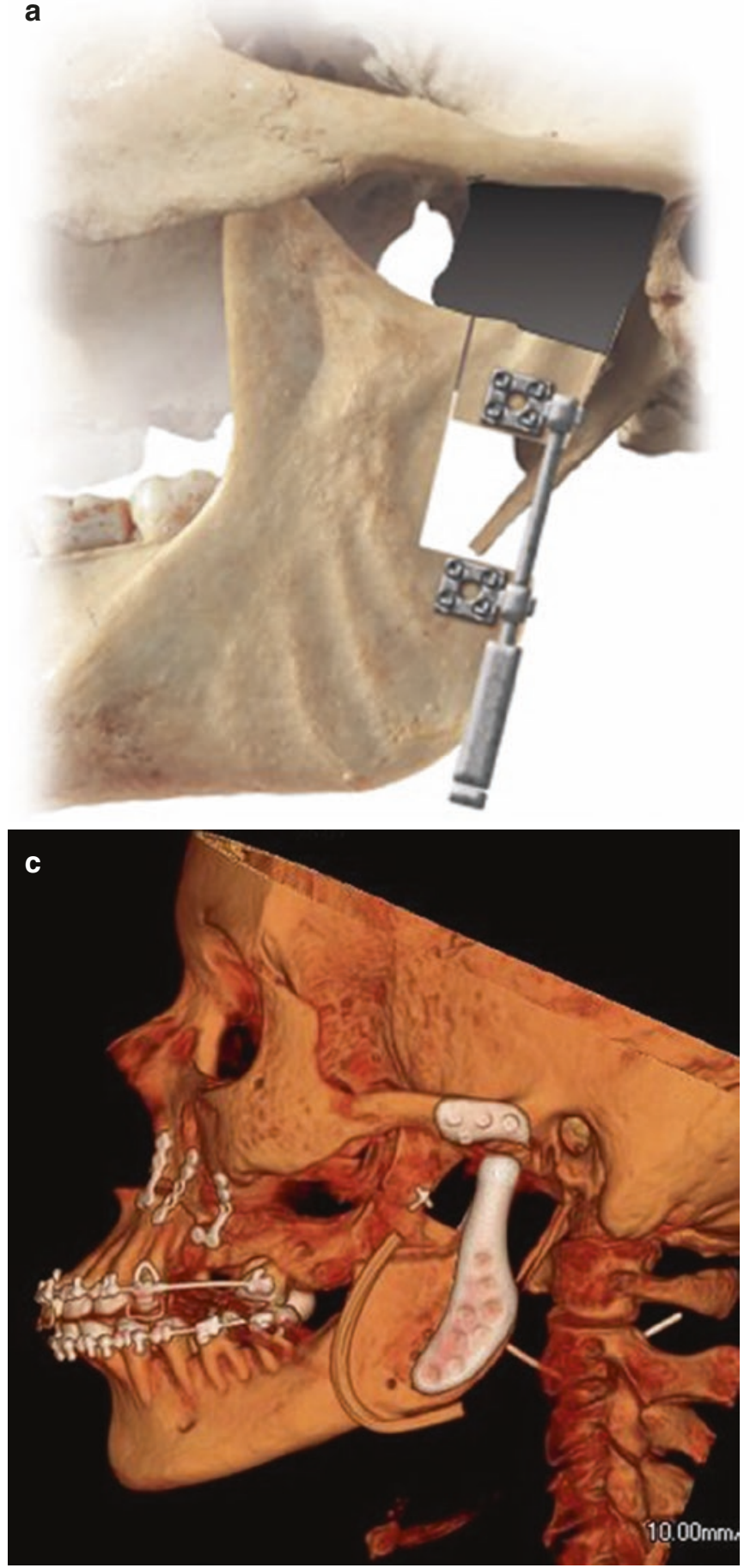

CAssociation of Oral and Maxillofacial Surgeons of India b

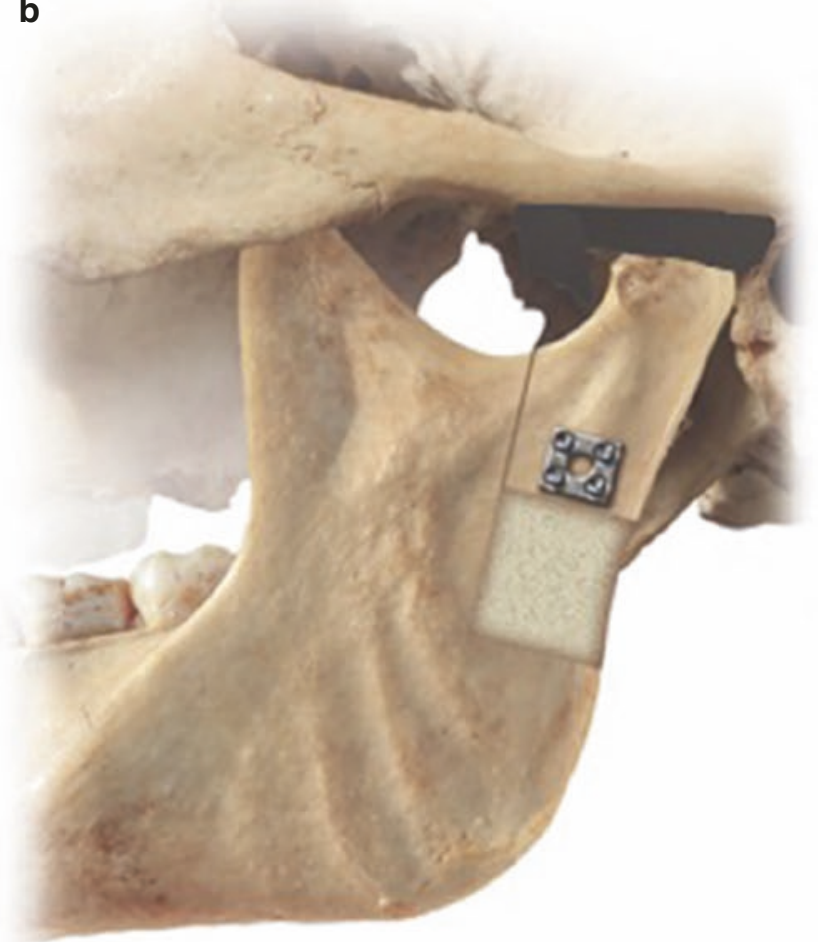

Fig. 65.11 (a) Transport distraction osteogenesis, (b) shows woven bone formation in place of callus in distracted gap, (c) alloplastic TMJ replacement with simultaneous release and OGS

\subsubsection{Alloplastic Total TMJ Replacement (TJR) (Fig. 65.11c)}

The alloplastic joint consists of a fossa component and a ramal component, as a stock or a custom-made prosthesis. Re-ankylotic scar tissue in an adult is best treated by total alloplastic TM joint replacement. Dongmei He and Yi Hui
$\mathrm{Hu}$ reported that alloplastic TMJ prosthesis is a reliable method to treat recurrent TMJ ankylosis. It may be accompanied by bimaxillary osteotomies for simultaneous correction of secondary ankylotic deformity as well. Its distinct disadvantage is the fact that if used in children, it would need to be replaced [42]. 
Mercuri et al. [43] specified the indications of TJR (Box 65.2).

Based on all the above RCU reconstruction options, guidelines are proposed for technique of RCU reconstruction in various clinical situations (Table 65.3).

\section{Box 65.2}

1. Recurrent fibrous or bony ankylosis not responsive to the modalities of treatment which have been hitherto applied

2. Failed (bone and soft) tissue grafts

3. Loss of vertical mandibular height and occlusal relationship due to bone resorption, trauma, developmental abnormalities, or pathological lesions

4. Severe inflammation of TMJ involving damage to its structures and lack of response to other treatment methods.

Table 65.3 Guidelines for technique of RCU reconstruction in various clinical situations

\begin{tabular}{|c|c|c|c|c|}
\hline & Situations & & Technique & Justification \\
\hline \multirow[t]{2}{*}{1.} & \multirow[t]{2}{*}{$<8$ years } & $\begin{array}{l}\text { (a) With facial } \\
\text { asymmetry }\end{array}$ & $\begin{array}{l}\text { Costochondral } \\
\text { Graft } \pm \text { eventual } \\
\text { high } \\
\text { condylectomy }\end{array}$ & $\begin{array}{l}\text { - Potential for } \\
\text { growth } \\
\text { - Treat } \\
\text { overgrowth } \\
\text { like condylar } \\
\text { hypertrophy }\end{array}$ \\
\hline & & $\begin{array}{l}\text { (b) Without } \\
\text { facial } \\
\text { asymmetry }\end{array}$ & Coronoid graft & $\begin{array}{l}\text { Potential for } \\
\text { growth not } \\
\text { required } \\
\text { - Cannot do } \\
\text { ramus } \\
\text { osteotomy } \\
\text { because of } \\
\text { inadequate } \\
\text { ramal width }\end{array}$ \\
\hline \multirow[t]{2}{*}{2.} & \multirow[t]{2}{*}{$>8$ years } & $\begin{array}{l}\text { (a) With } \\
\text { prominent } \\
\text { antegonial } \\
\text { notch }\end{array}$ & $\begin{array}{l}\text { Vertical ramus } \\
\text { osteotomy }\end{array}$ & $\begin{array}{l}\text { - Reduces } \\
\text { antegonial } \\
\text { notch }\end{array}$ \\
\hline & & $\begin{array}{l}\text { (b) Without } \\
\text { prominent } \\
\text { antegonial } \\
\text { notch }\end{array}$ & $\begin{array}{l}\text { L-shaped ramus } \\
\text { osteotomy }\end{array}$ & $\begin{array}{l}\text { - Maintains } \\
\text { height of ramus } \\
\text { - Adequate } \\
\text { ramal size }\end{array}$ \\
\hline \multirow[t]{2}{*}{3.} & \multirow[t]{2}{*}{ Re-ankylosis } & (a) Child & Ramal distraction & $\begin{array}{l}\text { - Free graft } \\
\text { will not take } \\
\text { in scar tissue } \\
\text { - Alloplastic } \\
\text { joint will not } \\
\text { grow }\end{array}$ \\
\hline & & (b) Adult & $\begin{array}{l}\text { Total alloplastic } \\
\text { TMJ replacement }\end{array}$ & $\begin{array}{l}\text { Best option } \\
\text { for scarred } \\
\text { tissue }\end{array}$ \\
\hline
\end{tabular}

Table 65.4 Treatment guidelines for different types of ankylotic deformities

Type I (presence of occlusal cant): treatment planning for both maxilla and mandible

1. Involved side is normal and other side is excess $(<\mathbf{6 ~ m m}$ deficiency)

(a) Differential maxillary impaction

(b) Mandibular ramus osteotomy

(i) Sagittal split osteotomy

(ii) Inverted L ramus osteotomy

(c) \pm Genioplasty

2. Involved side is deficient and other side is normal $(<6 \mathbf{~ m m}$ deficiency)

(a) Differential down fracture and bone grafting of maxilla

(b) Mandibular ramus osteotomy

(i) Sagittal split osteotomy

(ii) Inverted L ramus osteotomy

(c) \pm Genioplasty

3. 6-8 $\mathrm{mm}$ discrepancy in maxilla (6-8 $\mathbf{~ m m}$ in mandible)

(a) Simultaneous maxillomandibular distraction

4. 6-8 $\mathrm{mm}$ discrepancy in maxilla ( $>\mathbf{8} \mathbf{~ m m}$ discrepancy in mandible)

(a) Cant correction in maxilla

(b) Mandibular distraction as per type II-4, 5, 6

(c) Simultaneous OGS with TJR

Type II: (with minimal occlusal cant) treatment planning for mandible alone

1. Mandibular ramus osteotomy

2. Genioplasty

3. Orthomorphic osteotomy

4. Orthomorphic distraction

5. Unidirectional distraction

6. Bidirectional distraction

7. Simultaneous OGS with TJR

\subsection{Facial Deformity Secondary to TMJ Ankylosis}

TMJ ankylosis leads to mandibular deformity of varying magnitude and morphology in the form of decreased ramus and/or body length, deviation of chin, retrognathic/microgenia, bird face deformity, and narrow PAS leading to OSA (refer Fig. 68.18). Hence, establishing a single set treatment protocol for all clinical situations is not possible. Table 65.4 is an attempt to classify and thereby establish treatment guidelines for different types of ankylotic deformities.

Surgical Techniques and Indications:

\subsubsection{Osteotomies (Fig. 65.12a-c)}

They are indicated for movements of about $6-8 \mathrm{~mm}$. For maxilla, Le Fort osteotomies and, for mandible, sagittal split osteotomy or inverted L ramus osteotomy may be performed.

\subsubsection{Le Fort I Osteotomy (Refer Chap. 69)}

It allows correction in three dimensions including roll, pitch, and yaw. For vertical maxillary excess, differential maxillary 

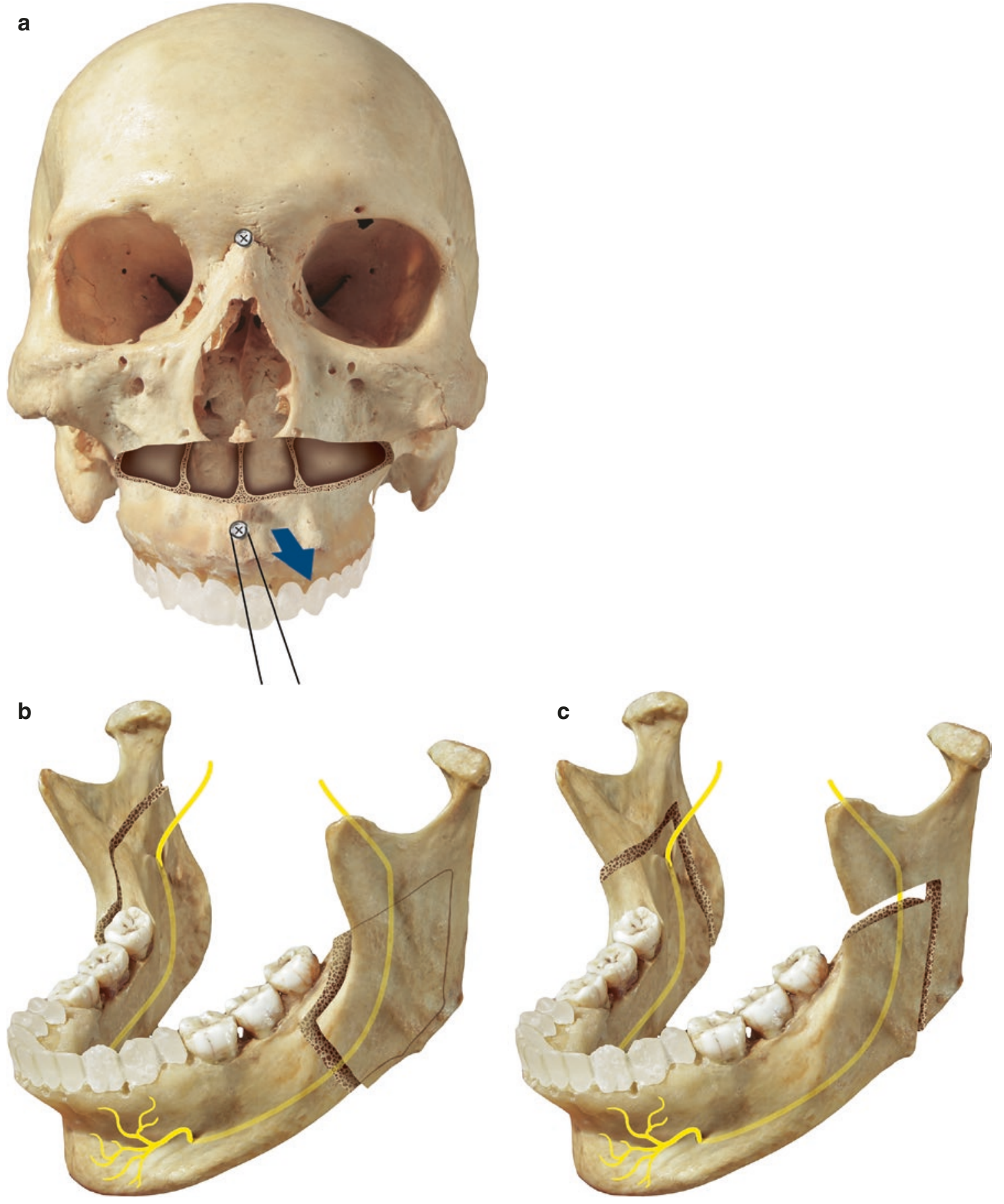

(C)Association of Oral and Maxillofacial Surgeons of India

Fig. 65.12 (a) Le Fort I osteotomy, (b) sagittal split osteotomy (SSO), (c) inverted L ramus osteotomy (ILRO) 
impaction may be performed, while for vertical maxillary deficiency, maxillary down fracture may be done along with bone grafting. Posterior maxillary height adjustments may also be required.

\subsubsection{Sagittal Split Osteotomy (SSO) (Refer Chap. 68)}

SSO is useful to differentially settle the occlusion according to the movement of the maxilla, as well as bring the retrognathic mandible forward. The procedure involves three corticotomies - the lingual horizontal, the buccal vertical, and the intermediate cuts. This osteotomy is generally done on the normal side in case of unilateral ankylosis. On the affected side, ramal contour and condylar anatomy distortions, reduced height of the ramus and steep mandibular plane angle make SSO difficult to perform.

\subsubsection{Inverted L Ramus Osteotomy (ILRO)}

(Fig. 68.6)

ILRO can be better utilized for increasing the ramal height. This technique is advocated in cases which require significant counterclockwise movement to increase projection of the lower third of the face when the ramal anatomy is altered.

The cuts are placed using a submandibular and/or an intraoral approach, posterior and superior to the inferior alveolar canal. A horizontal cut is made above and behind the lingula and then joined to the vertical cut from the inferior border of the mandible.

\subsubsection{Genioplasty (Figs. 68.29, 68.32 and 68.34)}

Patients indicated for genioplasty have normal ramal height, functional occlusion, deficient or normal pharyngeal airway space, and chin deviation or retrusion up to a maximum of 10 $\mathrm{mm}$, hence minimally deformed cases.

An extended genioplasty is performed with the osteotomy cuts extending up to the first molar on both sides. Advancement and translational movements in coronal and sagittal planes are carried out. Interpositional or onlay bone grafting may be done so as to achieve further symmetry wherever required.

Alloplastic chin implants made of PTFE, silicon, PEEK, or customized titanium are alternatives to the traditional genioplasty.

\subsubsection{Orthomorphic Osteotomy}

As opposed to conventional orthognathic surgery, an osteotomy used to restore morphology is termed "orthomorphic correction."

Patients who may undergo orthomorphic osteotomies are the ones having normal ramal height, functional occlusion, deficient or normal posterior airway space, and chin deviation or retrusion up to a maximum of $10 \mathrm{~mm}$, but this can be done only in cases of unilateral ankylosis. Most importantly, the primary indication for this surgery is the presence of soft tissue depression over the mandibular ramus on the unaffected side resulting in a contour defect. The osteotomy cuts are placed on the unaffected side.

Paul Salins [44] designed the osteotomy as an eccentric genioplasty extending from the body to the buccal cortex of the mandible. The cut changes from full thickness from the body to the sagittal buccal cortical cut in the ramus. The segment is then repositioned in such a way that it corrects the midline, the anteroposterior deficit, as well as the lateral morphological contour defects Fig. 65.13a.

\subsubsection{Distraction Osteogenesis (DO)}

Traditional bone grafting and orthognathic surgery have disadvantages - bony resorption, necrosis, donor site morbidity, limited advancement, relapse, etc.

Advancement of the mandible using SSO generally is restricted to a maximum of 10-12 mm. In ankylotic patients, the mandible requires much greater movements. Kohn et al. have reported high relapse after SSO [45], especially when used for advancement of mandible. Patients with ankylosis may report higher relapse rate than others. All the above issues may be dealt with DO.

Mandible lengthening with distraction leads to increase in bone stock of the mandible as well as proportionate and harmonious growth of the surrounding soft tissues. This bony regeneration is principally dependent on intramembranous ossification. Also healing and mineralization need adequate vascularity. Therefore, the incision to perform the surgery may be kept as limited as possible.

Early distraction may have beneficial effects, on the facial harmony as well as on the TMJ. If the anatomy and thereby function of the soft and hard tissues of craniofacial skeleton is established early in life, it may be possible to regain as near normal growth of the face.

Distraction in TMJ ankylosis patients with obstructive sleep apnea has led to decrease in snoring, daytime somnolence, Apnoea Hypopnoea Index (AHI), and oxygen desaturation episodes. The disadvantage however is that the device may be costly and needs to remain in situ for several months.

\subsubsection{Simultaneous Maxillomandibular DO}

This technique was first described by Molina and Monasterio in 1997 [46]. The first stage of surgery comprises of a Le Fort I osteotomy and a unilateral mandibular osteotomy. The distraction device is fixed to the mandible on the affected site. Under IMF, as the mandible moves forward, the maxilla comes downward, thereby correcting the cant and, simultaneously, the anteroposterior deficiency of the mandible. As this whole procedure is done under IMF, occlusion is main- 
tained. The second stage comprises of removal distraction device and an additional genioplasty if required.

This is indicated when the occlusal cant is such that there is vertical maxillary deficiency but with presence of functional occlusion. If the mandible alone is distracted, it may lead to occlusal disasters, hence the need to keep the patient under IMF while the distraction is being performed.

\subsubsection{Orthomorphic DO (Figs. 65.13a-d,} $78.23,78.24$ and 78.25$)$

As laid down by Manikandan et al. [47] , the concept of orthomorphic distraction of the mandible is a modification of Paul Salin's morphometric osteotomy. It involves the distraction of mandibular basal bone to correct the asymmetry in all dimensions, despite a deficient soft tissue capsule. It also provides more movement than that attainable via osteotomy.

The osteotomy design is similar to the one used in conventional orthomorphic surgery. Once the osteotomized segment is mobilized, an internal/external distractor may be placed. Maintenance of the periosteal attachment at the pterygomassetric sling region brings about a pivotal movement at the gonion facilitating the lateralization and advancement of the osteotomized segment. The mechanics of the mandibular asymmetry correction is based on the law of parallelogram of vectors.

\subsubsection{Genial Distraction}

Distraction of the chin may be performed either in isolation or simultaneously with ankylosis release. A case report on the latter has been published by Gunaseelan et al. [48] in 2007 wherein an extended genioplasty osteotomy was performed and external distractors placed and activated. They suggested that simultaneous genial distraction and arthroplasty may be performed in adults, as the mandible is no longer growing.

This is advocated in adults because they have functional stable occlusion. Therefore, distraction of the basal bone of the mandible without distracting the occlusion bearing segment will only correct the mandibular deformity and the OSAS, but not interfere with the occlusion. However, in children, dental relationships due to mandibular hypoplasia may be very severe and therefore this may not be the treatment of choice. Also, the eruption of the permanent teeth may interfere in osteotomy used in this technique.

Genial distraction is an option to gain larger advancements, although may be limited by an unsightly labiomental fold as advancement increases.

\subsubsection{Unidirectional DO (Fig. 65.14a-i)}

This procedure is indicated in patients with near normal ramal height but deficient corpus length, chin retrusion and deviation greater than $10 \mathrm{~mm}$, and presence of mild to moderate OSA.

For deficient corpus length, osteotomy cut is placed in the body region, generally between second premolar and first molar or between first and second molars. If the patient has not undergone presurgical orthodontics or if distraction is being performed pre-ankylosis release, the cut depends on the distance between the roots of the teeth and presence of impacted teeth. Maria B. Papageorge [49] advised that the cut should be placed in such a manner that the integrity of mandibular angle is maintained as it forms a very important aesthetic component of the face.

According to Samchukov, the vector of distraction should always be parallel to the occlusal plane, regardless of the direction of the osteotomy, to prevent anterior open bite deformity. Kunz et al. [50] has stated it to be a well-known complication of mandibular distraction. Unidirectional distraction is contraindicated in patients exhibiting vertical growth pattern. This is because unidirectional distraction in patients with a steep mandibular plane angle and substantially deficient ramal height may again lead to anterior open bite.

Internal distraction has a definite edge over external distraction in terms of aesthetics and patient compliance. However, chances of postoperative infection are greater with internal distractors. Also, they have limitations in terms of the amount of distraction possible.

Unidirectional distractors may be placed through either intraoral or extraoral approach. Surgical splints may be prepared on 3D-printed models preoperatively. Upon exposure of the bone, these premarked splints are positioned. Using them as guides, corticotomy sites and sites for distractor pins/screws are marked. Buccal corticotomy and lower border full-thickness cuts are placed. Distractor is fixed and the osteotomy cuts are completed. In case impacted teeth are in the way of the osteotomy cuts, they may be removed intraoperatively itself.

The distractors are activated to check completeness of the cuts and then brought back. Latency period is of 5-7 days, depending on the patient's age. Larger movements require larger periods of consolidation to prevent relapse.

After distracting for around 8-10 days, shape of the regenerate may be changed (callus molding) during the distraction process. Complications may include infection, tooth fracture, change of vector, loosening of pin, anterior open bite, and parotid fistula, but all can be managed conservatively. Callous molding can take care of occlusal discrepancies to a large extent, except that if there is absolutely no overjet. However, with some amount of overjet present, differential distraction may be done on the superior and inferior borders of the mandible. Herein, with the help of callous molding using IMF screws/eyelets and elastics, the inferior border may be distracted more than the superior border. 

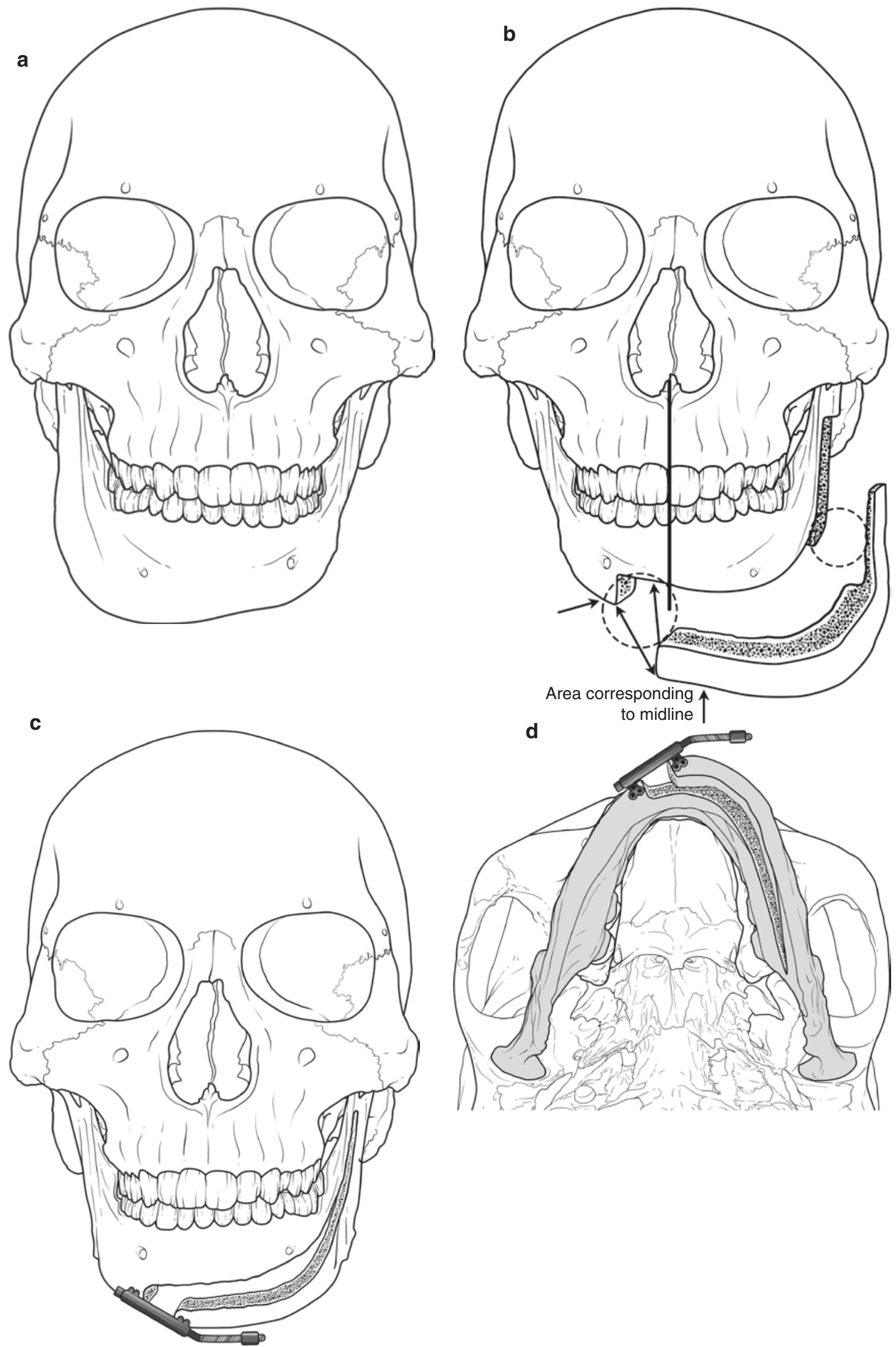

CAssociation of Oral and Maxillofacial Surgeons of India

Fig.65.13 (a, b) Orthomorphic osteotomy, (c, d) orthomorphic distraction 

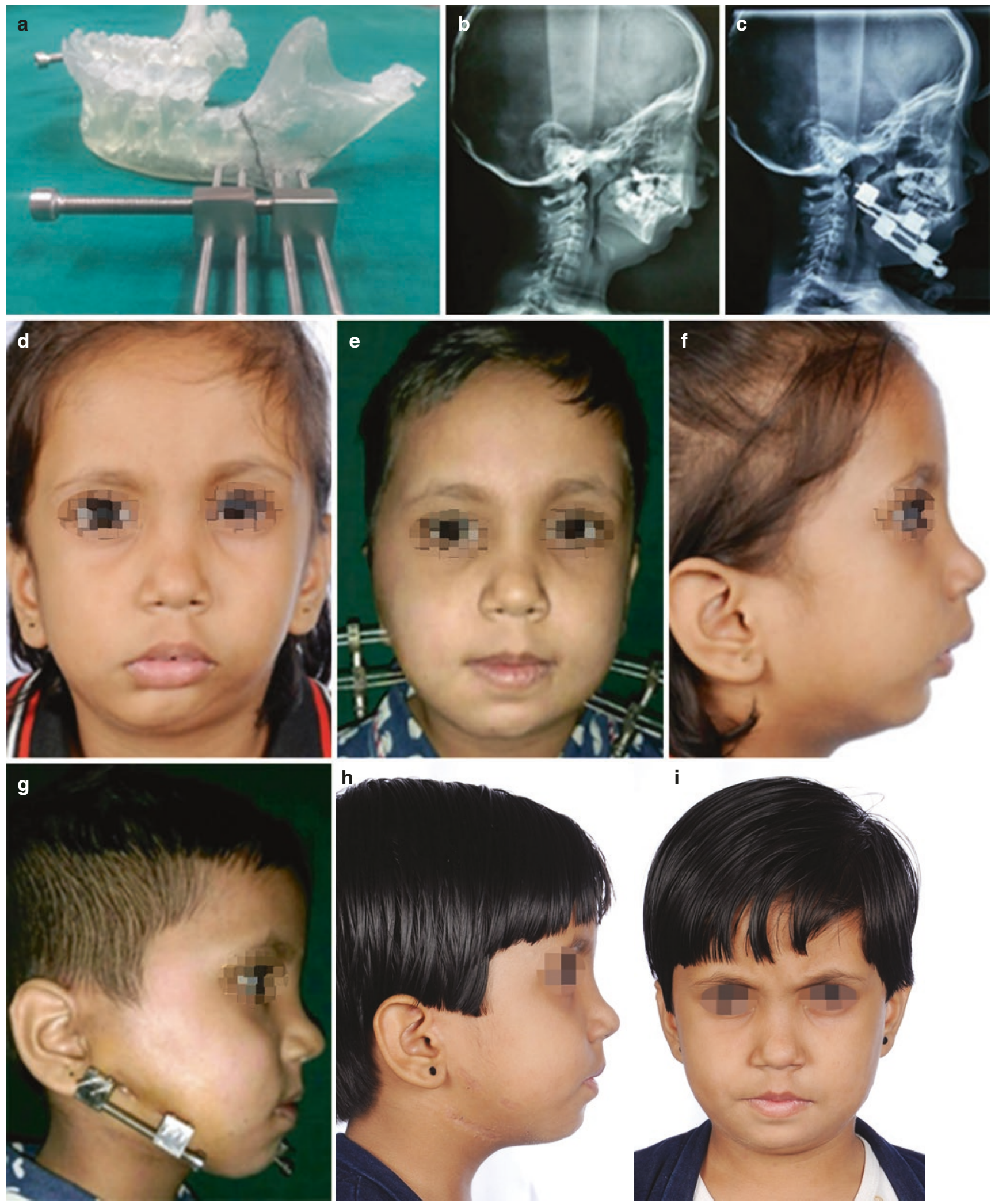

CAssociation of Oral and Maxillofacial Surgeons of India

Fig. 65.14 (a) Uniplanar distractor fixed on the 3D stereolithographic model with osteotomy cut marked, (b) preoperative lateral cephalogram, (c) post-distraction lateral cephalogram, (d) preoperative frontal view, (e) post-distraction, frontal view (f) preoperative lateral view, (g) post-distraction lateral view, (h) six-month follow-up lateral, (i) six month follow-up frontal 
Sometimes there may be occlusal locking thereby preventing the distracted regenerate from moving in the direction that is required. A composite block may be prepared on the tooth to first disocclude it and thereby get distraction in the favorable direction. Later occlusion may be regained by callous molding. The molding of the regenerate substantially reduces the time required for post operative orthodontics.

\subsubsection{Bidirectional DO (Fig. 65.15a-g)}

Bidirectional distractors are indicated for patients with severely shortened ramal height as well as corpal (body) length, requiring vertical as well as horizontal components of distraction. They are also indicated in cases with increased mandibular plane angle as well as for patients with moderate to severe OSA.

Surgical splints are prepared on 3D-printed models. Upon exposure of bone through a submandibular incision, two osteotomy cuts are placed on either side. One osteotomy cut is placed on the ramus and the other in the body region on either side of the angle, keeping the intermediate segment between 2.5 and $3 \mathrm{~cm}$ to avoid avascular necrosis. The device is secured to the mandible with six percutaneous pins (Schantz' pins)—one pair each at the ramus, angle, and corpus. The same procedure may be used for internal distractors as well.

The osteotomy site depends on position of teeth, lingula, mental foramen, and size of the intermediate segment. Vector of horizontal distraction should be parallel to occlusal plane, and vector of vertical distraction should be parallel to posterior border of ramus. In bilateral cases, horizontal and vertical components of either side should be parallel to each other. A 2-3 mm overcorrection is generally advisable. Callous molding may be used for occlusal adjustments. A single osteotomy cut may however be used for 3D or multivector distractors, thereby considerably reducing the surgical difficulty as well as the intraoperative time. Their main drawback is their prohibitive cost.

\subsubsection{Impact of Mandibular DO on OSA}

The ankylotic retruded mandible has an inefficient genial muscular apparatus as well, thereby causing retroglossal airway obstruction, therefore, OSAS. Mandibular advancement
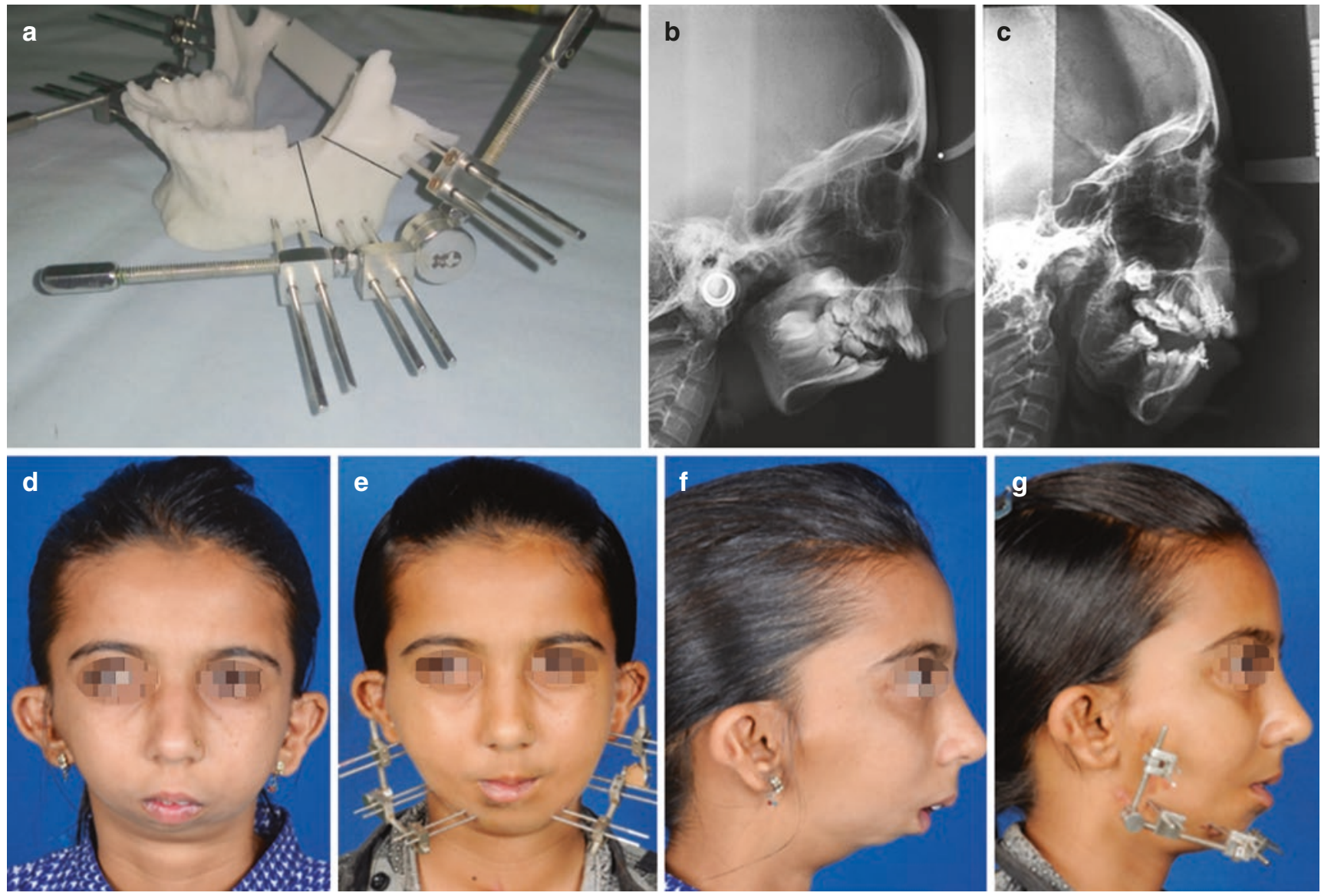

CAssociation of Oral and Maxillofacial Surgeons of India

Fig. 65.15 (a) Biplanar distractor fixed on the 3D stereolithographic model with osteotomy cuts marked, (b) preoperative lateral cephalogram, (c) post-distraction lateral cephalogram (marked increase in PAS

can be appreciated) (d) preoperative frontal view, (e) post-distraction frontal view, (f) preoperative lateral view, (g) post-distraction lateral view 
in such cases has shown $97 \%$ success in pediatric and $100 \%$ success in adult patients. Decrease in OSAS may be evaluated by an AHI less than 50\% decrease in the AHI with a near normal peripheral oxygen saturation.

\subsection{Sequence of Release and Deformity Correction}

OSA, in terms of AHI and PAS, is the principal factor that determines the sequence of release and deformity correction, i.e., whether to release first or distract first (refer Chap. 71 on OSAS and Figs. 71.8, 71.9, 71.10, 71.11, 71.12, 71.13 and 71.14).

\section{Single-Stage vs. Multistage Procedure:}

Treatment protocols can generally be categorized into singlestage or multistage treatments. Single-stage treatments institute ankylosis release and correction of secondary deformities (in the form of maxillary and mandibular osteotomies or distraction) all together. In the multistage protocol, ankylosis is released, and deformities are corrected in different stages.

\subsubsection{Multistage Surgical Treatment}

Severe secondary deformities are candidates for multistage procedures as a single-stage surgery may cause severe malocclusion. Correction of the same would require unrealistic amount of tooth movement.

Advantages: The multistage protocol is generally better for patients with significant medical comorbidity. This is so because a single-stage procedure obviously demands greater surgical time, thereby increasing the intraoperative risks. Multistage procedures facilitate early postoperative physiotherapy and, thus, a more stable postsurgical outcome. Also, the relapse monitoring is easier.

Disadvantages: Multiple surgeries, increased cost, and patient compliance issues

\subsubsection{Multistage with Ankylosis Release First}

According to López et al. [51], mandibular distraction should be performed after ankylosis release, as only then would be complete growth potential of the mandible be known. Releasing the ankylosis first would ensure adequate food intake, preparing the patients for subsequent, more complex surgeries. Also, surgeons could then formulate customized treatment plans based on individual clinical requirements and thereby provide more accurate correction of the deformity.

\subsubsection{Multistage with Distraction First}

Some surgeons recommend that distraction may be first used to restore the length and projection of the mandible. Later arthroplasty may be followed by orthognathic surgery to obtain mouth opening and settle the occlusion.

Advantages: The mandibular bone deficiency and the airway volume are both increased simultaneously. The subsequent intubation is much safer. Secondly, for patients with OSA, this treatment sequence reduces the incidence of postoperative airway issues-if ankylosis is released first, as the mandible falls back specially in bilateral cases, the already reduced airway space then gets further reduced, requiring reintubation or emergency tracheostomy. This incident can be avoided if distraction proceeds release.

\subsubsection{Single-Stage Surgical Treatment}

Patients with mild to moderate dental and skeletal deformities are ideal candidates for single-stage procedures. The minimal malocclusion may be treated by orthodontics alone. In these such cases, ankylosis release along with facial aesthetic surgeries which do not involve the occlusion may be performed, e.g., genioplasty, mandibular recontouring, and alloplastic implants.

Advantages: This corrects the restricted mouth opening as well as the secondary deformity simultaneously and avoids further surgeries. It provides low cost, immediate satisfaction, particularly in patients with psychological issues, and mild OSA.

Disadvantages: Maxillomandibular fixation often required after orthognathic surgery interferes with postoperative mouth opening exercises and therefore increases the chances of re-ankylosis. In case of internal distraction devices, there still arises the need for a second surgery to remove it. Also, the effect of distraction may be unsatisfactory because of the unstable condyle - if the osteotomy is placed in the ramus, the proximal segment may go up into the gap created after ankylotic mass removal, cause loss of vector and thereby re-ankylosis. Thus, to prevent this complication, while performing concomitant release and distraction, osteotomy cut may be placed only anterior to the angle, in the body region.

Another potential complication is the interference between physical exercises and distraction. A pseudo-joint may be formed in the callus area when the patient exercises, with the mouth opening force being directed toward the callus instead of the newly released joint. To prevent this complication, intensive active mouth opening exercise with wooden sticks/ heisters may be started from postoperative day 1 itself. Mouth 
opening exercise from day 7 onward, i.e., once distraction is started, should exclude the use of the above.

\subsubsection{Single-Stage Release with Orthognathic Surgery (OGS)}

As mentioned earlier, in patients that exhibit mild dental and skeletal deformities, aesthetic surgeries not involving the tooth-bearing segments may be performed.

For the patients with moderate skeletal deformities, SSO along with ILRO and bone grafts may be used to lengthen the affected ramus. If necessary, Le Fort I osteotomy may be used to correct the occlusal cant in unilateral cases or bring the posterior maxilla down in bilateral cases.

Songsong Zhu [52] performed a study on 27 adult patients who underwent simultaneous ankylosis release and correction of secondary deformity as a single surgery. No relapse occurred.

Yi Hui $\mathrm{Hu}$ [42] studied 11 patients where CAD/CAM technique was utilized as a guide to determine level of removal of ankylotic mass and placement of TMJ prosthesis. Simultaneous Le Fort I osteotomy was performed in two patients guided by digital templates. Results showed that alloplastic TMJ prosthesis is a reliable treatment for recurrent ankylosis. The CAD/CAM technique produces accurate therefore stable results.

Many cases would require a custom-made prosthesis, typically fabricated with a stereolithographic model. Ryu et al. in 2016 reported a case where bilateral TM joints were replaced with a custom-made TMJ prosthesis. Virtual surgical planning (VSP) and STL model simulation helped in placing the mandible in a new improved occlusal and aesthetic profile [53]. This has become a routine procedure now in adult patients, i.e., custom-made joints based on VSP along with simultaneous OGS.

\subsubsection{Single-Stage Release with DO}

Simultaneous interpositional arthroplasty with DO for TMJ ankylosis, apart from increasing the length of the mandible, leads to the correction of gross facial asymmetry and midline shift and provides space for eruption of hitherto unerupted teeth. It helps reduce treatment time and need for an additional surgery and also alleviates the need for second tracheostomy, if the fiberoptic intubation is not possible.

Only unidirectional distraction in the mandibular body (in patients with AHI 15-30) may be performed along with release. If bidirectional distraction is performed, after placing the arthroplasty cuts, if two more cuts are placed in the body and the ramus, there are increased chances of avascular necrosis of the segments. Thus, bidirectional distraction may not be performed along with release; it may be performed either pre- or post-release, unless a $3 \mathrm{D}$ distractor is being used.

Krishna Rao et al. [54] performed a study on six patients and concluded that simultaneous release with DO should be used to correct ankylosis with facial asymmetry and reported satisfactory cosmetic correction of deformities. Girish B Giraddi et al. [55] performed a similar study with nine cases and concluded that it not only corrects the facial deformity but also saves the trauma of a second difficult intubation.

Lopez and Dogliotti [51] in 2004 asked a pertinent question- "Is it reasonable to perform ankylosis release and mandibular distraction simultaneously without knowing which patients will be able to experience growth with time? In that case it would be necessary to predict growth to apply the exact amount of mandibular distraction for obtaining stable results." This question can now be answered-in children, the only indications for simultaneous release with distraction would be moderate to severe OSA, with AHI 15-30 (however with normal ramal height) and extreme facial deformity causing issues with the child's psyche.

\subsubsection{Single-Stage Release with Genioplasty}

Most literature till date has either been looking at ankylosis in isolation from facial asymmetry and has been dealing with only ankylosis release or facial deformity as staged events or has been looking at correcting one of the biggest facial deformities, all at one go. There is no middle path mentioned in literature wherein ankylosis and a substantial part of its related deformities can be corrected without the following problemsa supra-major surgery under general anesthesia, extremely expensive 3D-printed splints and alloplastic joint replacements, expensive and protracted orthodontics requiring multiple hospital visits from the underprivileged, undernourished patients living in far-flung areas, and long-term patient noncompliance for distraction. Also, if osteotomies are performed before the TMJ attains stability, surgical outcome may be less than satisfactory as related to aesthetics and occlusion.

A suggested solution for the above problem in adults with mild to moderate OSA is a single-stage surgery-ankylosis release by interpositional arthroplasty simultaneously with genioplasty. It would provide a one-step solution to restoration of function and reasonable aesthetics within a reasonable amount of general anesthesia time, no occlusal discrepancies, no compliance for distraction, and no need for orthodontics. Most importantly, it would not interfere with any other secondary corrective procedure later, if the patient so desires. 
The author has conducted a study on 43 ankylosed joints \& set forth single-staged treatment guidelinesankylosis release, RCU reconstruction, and extended advancement centering genioplasty for increase in mouth opening, restoration of ramal height, and improvement in facial asymmetry as well as OSA. Average mouth opening at maximum follow-up of 20 months was $34.36 \mathrm{~mm}$ with no reported recurrence. Average increase in $\mathrm{N}$ perpendicular to Pog was $7.16 \mathrm{~mm}$, average decrease in neck-chin angle and labiomental angle was $31.6^{\circ}$ and $35.4^{\circ}$; respectively, average increase in PAS was $2.92 \mathrm{~mm}$. Average 50\% improvement of AHI was seen in all 18 patients who had OSA [56].

\subsection{Guidelines for sequencing of release and deformity correction}

\subsubsection{In Ankylosed Patients: To Decide Regarding Distraction Pre-/Post-/ Simultaneously with Release (Table 65.5)}

- AHI >30: Children and adults—distract first

- $\mathrm{AHI}<15$

- Children

Release first

Simultaneous release and unidirectional distraction

- Adult

Release first

Simultaneous release with/without joint replacement and osteotomies

Simultaneous release and unidirectional distraction

- AHI 15-30

- Short ramus (requiring bidirectional distraction) Children and adults: distract first

- Normal ramus (requiring unidirectional distraction) Children: simultaneous release and distraction Adults

- Joint replacement with osteotomies

- Simultaneous release with distraction

\subsubsection{In Released Patients: To Decide Regarding Type of DO}

- Only body deficiency with normal Mandibular Plane Angle (MPA) - unidirectional distraction

- Body and ramus deficiency with steep MPA-bidirectional distraction

- AHI $>30$ - bidirectional distraction

- Asymmetry of mandibular angles-one side unidirectional, one side bidirectional distraction

- Dysmorphic asymmetry: orthomorphic distraction

- Functional occlusion in adults with normal ramus: genial distraction

\subsection{Unfavorable Results in TMJ Ankylosis Surgery (Table 65.6)}

\section{Table 65.6}

1. Issues in primary surgery

(a) Incomplete appreciation of the extent of the deformity

(i) Unilateral or bilateral

(ii) Length of the coronoid process

(b) Anaesthesia-related issues

(i) Excessive bleeding during fibreoptic intubation

(ii) Emergency tracheostomy

(c) Intra- and immediate postoperative issues

(i) Surgical mishaps

1. Nerve traction

2. Inadequate exposure of the bony block

3. Bleeding from pterygoid venous plexus

4. Injury to the internal maxillary artery

(ii) Failure to achieve adequate mouth opening

1. Ipsilateral joint release $\rightarrow$ ipsilateral coronoidectomy $\rightarrow$ contralateral intraoral coronoidotomy

(iii) Severe bradycardia on mouth opening

(iv) Postoperatively open bite after bilateral arthroplasty/ coronoidectomy

(d) Intermediate and long-term issues following primary surgery

(i) Re-ankylosis-reasons

1. Incomplete or inadequate primary release

2. Inadequate mouth opening exercises

(ii) Growth alterations of costochondral graft

2. Issues related to secondary surgery of deformities

(a) No control of movement of proximal segment

(b) Careful calculation of the vector

Table 65.5 Relation of Apnea-hypoapnea index with PAS

\begin{tabular}{|c|l|l|}
\hline PAS $(\mathrm{mm})$ & AHI & Rating \\
\hline$>8$ & $<5$ & Normal \\
\hline 8 & $5-10$ & Mild sleep apnea \\
\hline $5-7$ & $15-30$ & Moderate sleep apnea \\
$<4$ & $>30$ & Severe sleep apnea \\
\hline
\end{tabular}

\subsection{Conclusion}

- Traditionally, TMJ ankylosis and its associated micrognathia have been treated in multiple stages. Rehabilitation of mandibular function, prevention of re-ankylosis, and promotion of mandibular growth have been the main aims of treatment. The treatment has now advanced from only 
ankylosis release to total joint replacement as well as facial deformity correction, all simultaneously as far as possible.

- The patient at the time of presentation itself is looked at holistically. A more nuanced decision tree for treatment planning is formulated, be it for release, new joint reconstruction, distraction, osteotomies, or the sequencing of release vs. facial deformity correction. The most critical deciding factor in the treatment planning is obstructive sleep apnea, and the key for successful treatment is appropriate planning at each stage.

- Therefore, just because in the past, we were releasing all ankylosis first and doing corrective surgeries later, we cannot continue doing the same; albeit neither can we distract all patients first because that is the latest fad. An extensive review of literature as well as the vast experience of the author has helped lay down guidelines for most clinical scenarios of varying magnitude of deformities in TMJ ankylosis.

- In a few patients, some degree of facial imbalance may continue to persist even after surgical interventions. Additional aesthetic surgery, such as malarplasty reshaping of the chin, angle and lower border of the mandible, may be required to achieve the best results [57].

- Since TMJ ankylosis is generally a disease of the underprivileged and socioeconomically backward strata of society, it should be noted that the deformity cannot be the only criteria to select the surgical procedure. Patient's preference, the treatment cost, and compliance also need to be taken into consideration. Some patients are more concerned about their mouth opening and aesthetics rather than their occlusal disharmony.
Acknowledgment I wish to convey my deepest thanks and sincerest gratitude to my resident Dr. Hrushikesh Gosai, without whose observations, comments, and technical expertise this manuscript would have not been possible. A mere word of thanks is not sufficient to express the unflinching support and inestimable aid of my staff Dr. Jigar, Dr. Utsav, and Dr. Bipin and my residents Dr. Hiral, Dr. Philip and Dr. Nisha during the preparation of this chapter. I would be failing in my duty if I did not recognize the contribution of my biggest teacher in TMJ ankylosis, my Ex-Hod Dr. Babu Parmar.

Disclosure Authors have no financial conflicts to disclose

\subsection{Case Scenarios}

\section{Case 1 (Fig. 65.16a-h)}

A 23-year-old male patient presented with unilateral rightsided bony ankylosis with retruded and deviated chin with body and ramal length near normal. So, the patient was planned for advancement centering genioplasty and onlay bone graft along with TMJ ankylosis release.

\section{Case 2 (Fig. 65.17a-f)}

A 14-year-old male patient presented with facial asymmetry secondary to TMJ ankylosis with moderate OSA. Cephalometric analysis revealed deficiencies in the length of the mandibular ramus and corpus of the right side and the corpus alone on the left side. So, he was planned for biplanar distraction on right side and uniplanar distraction on left side. 

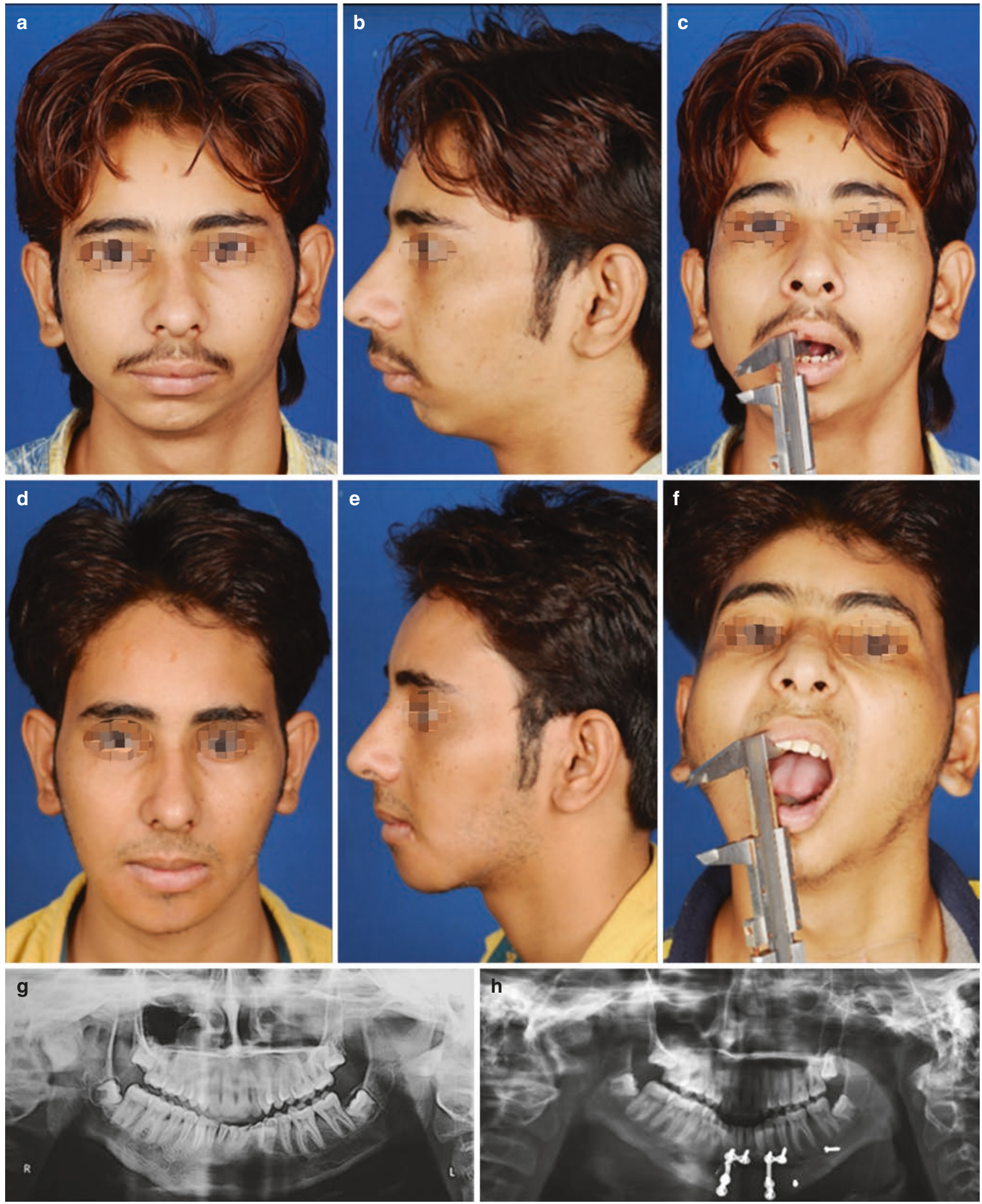

CAssociation of Oral and Maxillofacial Surgeons of India

Fig. 65.16 (a-h) Case 1 Right side TMJ ankylosis (a) Preoperative frontal view, (b) preoperative lateral view, (c) preoperative mouth opening, (d) postoperative frontal view, (e) postoperative lateral view, (f) postoperative mouth opening, (g) preoperative orthopantomogram, (h) postoperative orthopantomogram (note the genioplasty and onlay bone graft fixations) 

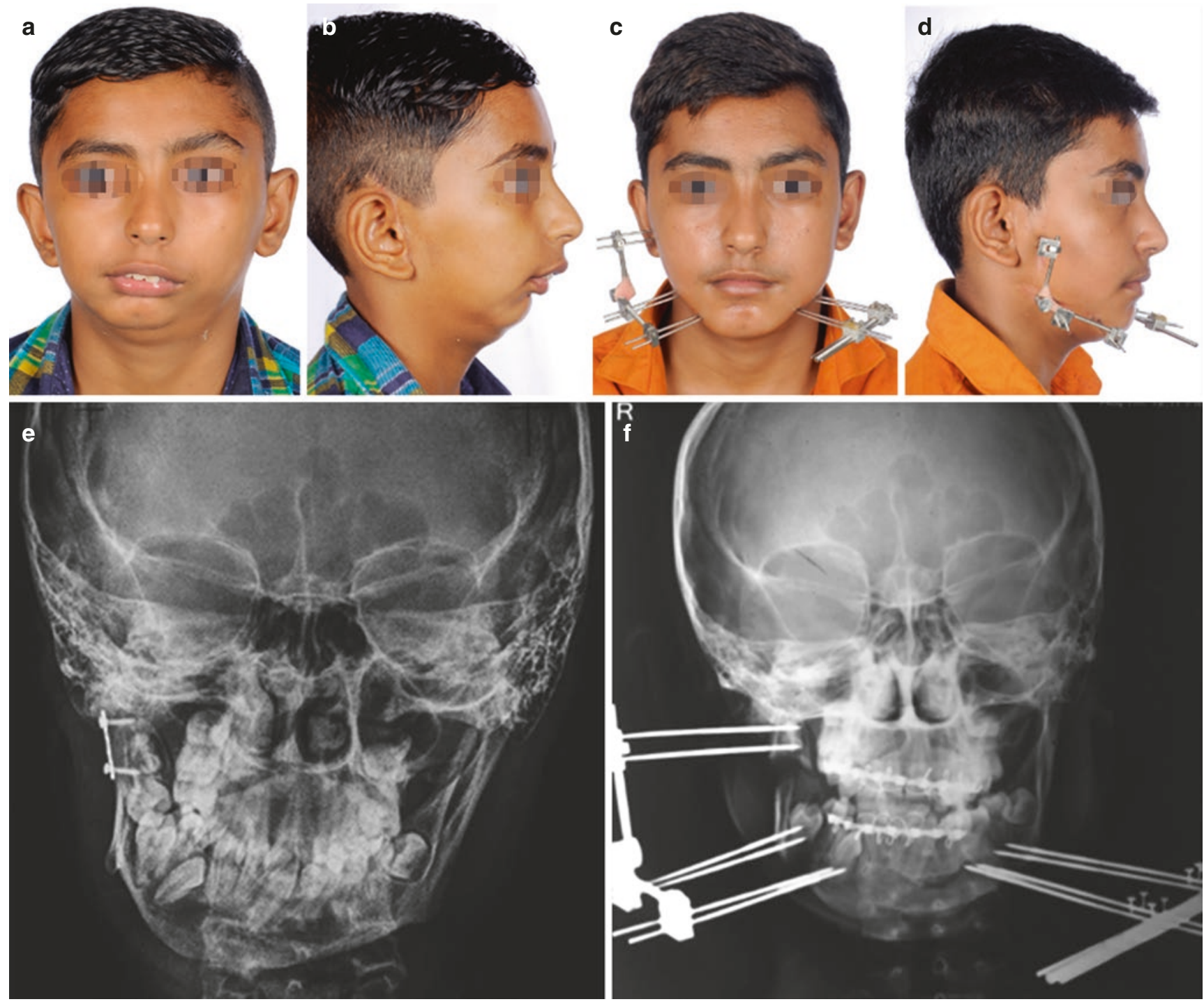

CAssociation of Oral and Maxillofacial Surgeons of India

Fig. 65.17 Case 2 (a) Preoperative frontal view, (b) preoperative lateral view, (c) postoperative frontal view, (d) postoperative lateral view, (e) Pre-distraction PA cephalogram, showing implant on the right side

\section{References}

1. Sporniak-Tutak K, Janiszewska-Olszowska J, Kowalczyk R. Management of temporomandibular ankylosis-compromise or individualization-a literature review. Med Sci Monit. 2011;17(5):RA111-6.

2. Kaban LB, Perrott DH, Fisher K. A protocol for management of temporomandibular joint ankylosis. J Oral Maxillofac Surg. 1990;48:1145-51.

3. Sawhney C. Bony ankylosis of the temporomandibular joint: follow-up of 70 patients treated with arthroplasty and acrylic space interposition. Plast Reconstr Surg. 1986;77:29-38.

4. Yan Y-B, Liang S-X, Shen J, Zhang J-C, Zhang Y. Current concepts in the pathogenesis of traumatic temporomandibular joint ankylosis. Head Face Med. 2014;10:35.

5. Mehrotra D, Sidebottom AJ. Management of ankylosis of the temporomandibular joint. In: Brennan P, Schliephake H, Ghali GE, from the earlier surgery for interposition arthroplasty, (f) postoperative PA cephalogram

Cascarini L, editors. Maxillofacial surgery. 3rd ed. Edinburgh: Churchill Livingstone; 2017.

6. Aziz SR. Andy Gump and his deformity. J Oral Maxillofac Surg. 2010;68(3):651-3.

7. Anantanarayanan P, Narayanan V, Manikandhan R, Kumar D, et al. Int J Pediatr Otorhinolaryngol. 2008;72:385-9.

8. Andrade NN, Raikwar KR. Management of patients with obstructive sleep apnoea induced by temporomandibular joint ankylosis: a novel 2-stage surgical protocol and report of 5 cases. Asian J Oral Maxillofac Surg. 2009;21:27-32.

9. Wu XG, Hong M, Sun KH. Severe osteoarthrosis after fracture of the mandibular condyle: a clinical and histologic study of seven patients. J Oral Maxillofac Surg. 1994;52(2):138-42.

10. Dhasmana S, Singh V, Pal US. Awake blind nasotracheal intubation in temporomandibular joint ankylosis patients under conscious sedation using fentanyl and midazolam. J Maxillofac Oral Surg. 2010;9(4):377-81. 
11. Quinn PD, Granquist EJ ; Surgical approaches to temporomandibular joint; In: Quinn PD, Granquist EJ (eds) Atlas of temporomandibular joint surgery 2 Wiley Chichester 2015, 31-55.

12. Al-Kayat A, Bramley P. A modified pre-auricular approach to the temporomandibular joint and malar arch. Br J Oral Surg. 1979;17:91-103.

13. Miloro M, Redlinger S, Pennington DM, Kolodge T. In situ location of the temporal branch of the facial nerve. J Oral Maxillofac Surg. 2007;65:2466-9.

14. Talebzadeh N, Rosenstein TP, Pogrel MA. Anatomy of the structures medial to the temporomandibular joint. Oral Surg Oral Med Oral Pathol Oral Radiol Endod. 1999;88:674-8.

15. Alexander RW, James RB. Postauricular approach for surgery of the temporomandibular articulation. J Oral Surg. 1975;33:346-50.

16. Kaban LB, Bouchard C, Troulis MJ. A protocol for management of temporomandibular joint ankylosis in children. J Oral Maxillofac Surg. 2009;67(9):1966-78.

17. Clarkson P, Kenshole HH. Esmarch's operation for the relief of ankylosis and of pain in the temporomandibular joint; a modified operative technique. Br Dental J. 1949;87:257-64.

18. Topazian RG. Etiology of ankylosis of temporomandibular joint: analysis of 44 cases. J Oral Surg Anesth Hosp Dent Serv. 1964:22:227-33.

19. Topazian RG. Comparison of gap and interposition arthroplasty in the treatment of temporomandibular joint ankylosis. J Oral Surg Am Dent Assoc. 1966;24:405-9.

20. Elgazzar RF, Abdelhady AI, Saad KA, Elshaal MA, Hussain MM, Abdelal SE, Sadakah AA. Treatment modalities of TMJ ankylosis: experience in Delta Nile, Egypt. Int J Oral Maxillofac Surg. 2010;39:333-42.

21. He Y, Huang T, Zhang Y, An J, He L. Application of a computerassisted surgical navigation system in temporomandibular joint ankylosis surgery: a retrospective study. Int J Oral Maxillofac Surg. 2017;46:189-97.

22. Ray PDA, Sundar Biswas DP, Chatterjee DA. Trismus-an old concept for successful treatment in the present era-case reports. IOSR J Dent Med Sci. 2016;15(9):12-6.

23. He D, Yang C, Chen M, Zhang X, Qiu Y, Yang X, Li L, Fang B. Traumatic temporomandibular joint ankylosis: our classification and treatment experience. J Oral Maxillofac Surg. 2011;69:1600-7.

24. Nitzan DW, Tair JA, Lehman H. Is entire removal of a posttraumatic temporomandibular joint ankylotic site necessary for an optimal outcome? J Oral Maxillofac Surg. 2012;70(12:e683-99.

25. Yang X, Lu C, Dong M, He D, Yang C, Hu Y. Evaluation of the condyle remodeling after lateral arthroplasty in growing children with temporomandibular joint ankylosis. Sci Rep. 2017;7:1-827.

26. Albert TW, Merrill RG. Temporalis myofascial for reconstruction of temporomandibular joint. Oral Maxillofac Surg Clin North Am. 1989;1:346.

27. Pogrel MA, Kaban LB. The role of a temporalis fascia and muscle flap in temporomandibular joint surgery. J Oral Maxillofac Surg. 1990;48:14-9.

28. Dimitroulis G. The interpositional dermis-fat graft in the management of temporomandibular joint ankylosis. Int J Oral Maxillofac Surg. 2004;33:755-60.

29. Thangavelu A, Thiruneelakandan S, Prasath $\mathrm{CH}$, Chatterjee D. Fate of free fat dermis graft in TMJ interpositional gap arthroplasty: a long term MRI study. J Maxillofac Oral Surg. 2015;14(2):321-6.

30. Gaba S, Sharma RK, Rattan V, Khandelwal N. The long-term fate of pedicled buccal pad fat used for interpositional arthroplasty in TMJ ankylosis. J Plast Reconstr Aesthet Surg. 2012;65:1468-73.

31. Akhter M, Ahmed N, Arefin MRU, Sobhan MU, Molla MR, Kamal M. Outcome of amniotic membrane as an interpositional arthroplasty of TMJ ankylosis. Oral Maxillofac Surg. 2016;20(1):63-71.
32. Gunaseelan R. Condylar reconstruction in extensive ankylosis of temporomandibular joint in adults using resected segment as autograft. A new technique. Int J Oral Maxillofac Surg. 1997;26:405-7.

33. Khadka A, Hu J. Autogenous grafts for condylar reconstruction in treatment of TMJ ankylosis: current concepts and considerations for the future. Int J Oral Maxillofac Surg. 2012;41:94-102.

34. Mehta G, Mohammad S, Ram H, Singh V, Chak RK, Garg S, Vishwakarma K. Re-assessment of coronoid as a graft for condylar reconstruction in TMJ ankylosis patients: a prospective study and literature review. J Maxillofac Oral Surg. 2017;16(2):175-80.

35. Liu Y, Li J, Hu J, Zhu S, Luo E, Hsu Y. Autogenous coronoid process pedicled on temporal muscle grafts for reconstruction of the mandible condylar in patients with temporomandibular joint ankylosis. Oral Surg Oral Med Oral Pathol Oral Radiol Endod. 2010;109(2):203-10.

36. Poswillo DE. Biological reconstruction of the mandibular condyle. Br J Oral Maxillofac Surg. 1987;25(2):100-4.

37. Salash JR, Hossameldin RH, Almarza AJ, Chou JC, McCain JP, Mercuri LG, Wolford LM, Detamore MS. Potential indications for tissue engineering in temporomandibular joint surgery. J Oral Maxillofac Surg. 2016;74:705-11.

38. Liu Y, Khadka A, Li J, Hu J, Zhu S, Hsu Y, Wang Q, Wang D. Sliding reconstruction of the condyle using posterior border of mandibular ramus in patients with temporomandibular joint ankylosis. Int J Oral Maxillofac Surg. 2011;40:1238-45.

39. Cheung LK, Zheng LW, Ma L, Shi XJ. Transport distraction versus costochondral graft for reconstruction of temporomandibular joint ankylosis: which is better? Oral Surg Oral Med Oral Pathol Oral Radiol Endod. 2009;108(1):32-40.

40. Sharma R, Manikandhan R, Sneha P, Parameswaran A, Kumar JN, Sailer H. Neocondyle distraction osteogenesis in the management of temporomandibular joint ankylosis: report of five cases with review of literature. Indian J Dent Res. 2017;28(3):269-74.

41. Xiao E, Zhang Y, An J, Li J, Long-term YY. Long-term evaluation of the stability of reconstructed condyles by transport distraction osteogenesis. Int J Oral Maxillofac Surg. 2012;41:1490-4.

42. Hu YH, Zhang L, He DM, Yang C, Chen MJ, Zhang SY, Li H, Ellis E. Simultaneous treatment of temporomandibular joint ankylosis with severe mandibular deficiency by standard TMJ prosthesis. Sci Rep. 2017;7:45271.

43. Movahed R, Mercuri LG. Management of temporomandibular joint ankylosis. Oral Maxillofac Surg Clin NA. 2015;27(1):27-35.

44. Salins PC, Venkatraman B, Kavarody M. Morphometric basis for orthomorphic correction of mandibular asymmetry. J Oral Maxillofac Surg. 2008;66:1523-31.

45. Kohn MW. Analysis of relapse after mandibular advancement surgery. J Oral Surg. 1978;36:676-84.

46. Ortiz Monasterio F, Molina F, Andrade L, Rodriguez C, Sainz Arregui J. Simultaneous mandibular and maxillary distraction in hemifacial microsomia in adults: avoiding occlusal disasters. Plast Reconstr Surg. 1997;100:852-61.

47. Parameswaran A, Ramanathan M, Pendem S, Jeyakumar N. Orthomorphic distraction-a new concept and its mechanics. J Cleft Lip Palate and Craniofac Anomal. 2013;4:1.

48. Gunaseelan R, Anantanarayanan P, Veerabahu M, Vikraman B. Simultaneous genial distraction and interposition arthroplasty for management of sleep apnoea associated with temporomandibular joint ankylosis. Int J Oral Maxillofac Surg. 2007;36:845-8.

49. Kunz C, Hammer B, Prein J. Manipulation of callus after linear distraction: a "lifeboat" or an alternative to multivectorial distraction osteogenesis of the mandible? Plast Reconstr Surg. 2000;105:674-9.

50. Papageorge MB, Apostolidis C. Simultaneous mandibular distraction and arthroplasty in a patient with temporomandibular joint ankylosis and mandibular hypoplasia. Case Rep. 1999;57(3):328-33. 
51. Lopez EN, Dogliotti PL. Treatment of temporomandibular joint ankylosis in children: is it necessary to perform mandibular distraction simultaneously? J Craniofac Surg. 2004;15:875-9.

52. Zhu S, Jiang Y, Pokhrel N, Hu J. Simultaneous correction of temporomandibular joint ankylosis and secondary dentofacial deformities in adult patients: surgical technique, treatment outcomes, and a consideration of the factors involved. J Craniofac Surg. 2015;26:2351-6.

53. Ryu J, Cho J, Kim HM. Bilateral temporomandibular joint replacement using computer-assisted surgical simulation and threedimensional printing. J Craniofac Surg. 2016;27:e450-2.

54. Rao K, Kumar S, Kumar V, Singh AK, Bhatnagar SK. The role of simultaneous gap arthroplasty and distraction osteogenesis in the management of temporo-mandibular joint ankylosis with mandibular deformity in children. J Craniomaxillofac Surg. 2004;5182:38-42.

55. Giraddi G, Arora K, Sai Anusha A. Distraction osteogenesis in the treatment of temporomandibular joint ankylosis with mandibular micrognathia. Ann Maxillofac Surg. 2016;6:68.

56. Anchlia S, Vyas SM, Dayatar RG, Domadia HL, Nagavadiya V. Guidelines for single-stage correction of TMJ ankylosis, facial asymmetry and OSA in adults. J Maxillofac Oral Surg. 2018;18(3):419-27.

57. Zhu S, Wang D, Yin Q, Hu J. Treatment guidelines for temporomandibular joint ankylosis with secondary dentofacial deformities in adults. J Cranio-Maxillofac Surg. 2013;41:e117-27.

Open Access This chapter is licensed under the terms of the Creative Commons Attribution 4.0 International License (http://creativecommons. org/licenses/by/4.0/), which permits use, sharing, adaptation, distribution and reproduction in any medium or format, as long as you give appropriate credit to the original author(s) and the source, provide a link to the Creative Commons license and indicate if changes were made.

The images or other third party material in this chapter are included in the chapter's Creative Commons license, unless indicated otherwise in a credit line to the material. If material is not included in the chapter's Creative Commons license and your intended use is not permitted by statutory regulation or exceeds the permitted use, you will need to obtain permission directly from the copyright holder. 